\title{
The Evolution and Role of the Saharan Air Layer during Hurricane Helene (2006)
}

\author{
SCOTT A. BRAUN \\ Mesoscale Atmospheric Processes Laboratory, NASA Goddard Space Flight Center, Greenbelt, Maryland \\ JASON A. SIPPEL \\ Morgan State University/NASA Goddard Space Flight Center, Greenbelt, Maryland \\ CHUNG-Lin SHIE \\ University of Maryland, Baltimore County, Baltimore, and NASA Goddard Space Flight Center, Greenbelt, Maryland \\ RYAN A. BOLLER \\ NASA Science Data Processing Branch, NASA Goddard Space Flight Center, Greenbelt, Maryland
}

(Manuscript received 23 January 2013, in final form 17 May 2013)

\begin{abstract}
The Saharan air layer (SAL) has received considerable attention in recent years as a potential negative influence on the formation and development of Atlantic tropical cyclones. Observations of substantial Saharan dust in the near environment of Hurricane Helene (2006) during the National Aeronautics and Space Administration (NASA) African Monsoon Multidisciplinary Activities (AMMA) Experiment (NAMMA) field campaign led to suggestions about the suppressing influence of the SAL in this case. In this study, a suite of satellite remote sensing data, global meteorological analyses, and airborne data are used to characterize the evolution of the SAL in the environment of Helene and assess its possible impact on the intensity of the storm. The influence of the SAL on Helene appears to be limited to the earliest stages of development, although the magnitude of that impact is difficult to determine observationally. Saharan dust was observed on the periphery of the storm during the first two days of development after genesis when intensification was slow. Much of the dust was observed to move well westward of the storm thereafter, with little SAL air present during the remainder of the storm's lifetime and with the storm gradually becoming a category-3 strength storm four days later. Dry air observed to wrap around the periphery of Helene was diagnosed to be primarily non-Saharan in origin (the result of subsidence) and appeared to have little impact on storm intensity. The eventual weakening of the storm is suggested to result from an eyewall replacement cycle and substantial reduction of the sea surface temperatures beneath the hurricane as its forward motion decreased.
\end{abstract}

\section{Introduction}

The role of the Saharan air layer (SAL) in modulating tropical cyclone genesis and intensity has received considerable attention recently. Early studies by Karyampudi and Carlson (1988) and Karyampudi and Pierce (2002) suggested that the SAL can have a positive influence on the growth of easterly waves and tropical cyclones through barotropic and baroclinic instabilities and by enhancing rising motion south of the SAL. More recent studies

Corresponding author address: Dr. Scott A. Braun, NASA GSFC, Mail Code 612, Greenbelt, MD 20771.

E-mail: scott.a.braun@nasa.gov focused on potential negative influences on storm development (Dunion and Velden 2004, hereafter DV04; Wu et al. 2006; Jones et al. 2007; Lau and Kim 2007a,b; Wu 2007; Dunion and Marron 2008; Sun et al. 2008, 2009; Reale et al. 2009; Shu and Wu 2009) and motivated field campaigns such as the Saharan Air Layer Experiment (SALEX; Rogers et al. 2006) and the National Aeronautics and Space Administration (NASA) African Monsoon Multidisciplinary Activities (AMMA) Experiment (NAMMA; Zipser et al. 2009).

In a key study to suggest a negative influence of the SAL on tropical cyclone genesis and evolution, DV04 hypothesized that the SAL negatively impacts tropical cyclones in the following ways: 1 ) it enhances a low-level

DOI: 10.1175/MWR-D-13-00045.1

(C) 2013 American Meteorological Society 
temperature inversion, maintained by radiative warming of dust, which suppresses deep convective development; 2 ) it strengthens African easterly jet (AEJ) easterlies and deep-layer vertical wind shear over the cyclone, which may inhibit storm intensification (e.g., Gray 1968; Merrill 1988; DeMaria and Kaplan 1994, 1999; Frank and Ritchie 1999, 2001; Rogers et al. 2003; Braun and Wu 2007); and 3 ) it promotes intrusions of dry air into tropical cyclones, which foster enhanced cold downdrafts (Emanuel 1989; Powell 1990) and lower the convective available potential energy in the storm core. Many subsequent studies often assumed, but did not clearly demonstrate, that the SAL's impacts were predominantly negative and were a major factor affecting hurricane activity in the Atlantic (e.g., Jones et al. 2007; Wu 2007; Sun et al. 2008, 2009; Shu and Wu 2009).

Other recent studies (Braun 2010a; Sippel et al. 2011) suggested that the impact of the SAL on tropical cyclones might be less than previously hypothesized. Braun (2010a) found little discernible negative impact of the SAL in a composite study using National Centers for Environmental Prediction (NCEP) Global Data Assimilation System (GDAS) Final Analyses. Using NCEP data from 41 storms that formed in the central and eastern Atlantic, he found few statistically significant differences in the composite structure of the SAL between tropical storms that intensified quickly in the days after formation and those that weakened. Thus, Braun concluded that the SAL was generally not a major determining factor in intensity change after tropical storm formation. Braun (2010a) also suggested that large-scale subsidence may be comparable to the SAL as a source of dry air in the tropical and subtropical Atlantic.

The NAMMA field campaign was focused on the transition of African continental convective systems to the maritime environment and the possible genesis of tropical cyclones. An important emphasis was the role of the SAL in genesis and intensification processes (Zipser et al. 2009). Zipser et al. (2009) suggested a possible negative role of the SAL in the cases of Tropical Storm (TS) Debby and Hurricane Helene, both of which were investigated during NAMMA. Ross et al. (2009) examined the African easterly waves (AEWs) that became Debby and Helene as well as a nondeveloping wave during NAMMA. Their results suggest that the AEJ (and by implication, the SAL) can have a positive or negative influence on wave growth via barotropic processes depending on the structure of the wave and the location of the jet maximum. Diabatic generation of potential vorticity south of the AEJ axis was also found to be a major contributor to development. Vizy and Cook (2009) showed evidence for suppression or delay of growth by the SAL in the development of Hurricane Ernesto, another NAMMA storm, compared to Debby. Reale et al. (2009) and Reale and Lau (2010) argued for the suppression of wave development by a warm and dry tongue of SAL air in the wave following Debby, although Braun (2010b) contended that Reale et al.'s interpretation of this feature as SAL air was incorrect, instead arguing that the warmth and dryness were the result of strong subsidence in a gap between two dust outbreaks.

Sippel et al. (2011) used ensemble forecasts to study factors affecting the intensity of TS Debby (2006). Their results suggested that dry SAL air delayed intensification during the very early stages of Debby, prior to formation of the tropical storm. However, the adjacent SAL air mass did not appear to affect the intensity at later times, when the storm was stronger. Among the ensembles, more intense cyclones were better able to shield themselves from entrainment of the dry, stable SAL air, as in Riemer and Montgomery (2011). Sippel et al. showed that ensemble members intensified most quickly while Debby was over relatively warm sea surface temperatures (SSTs), and more slowly when Debby was over cooler SSTs, suggesting that SSTs had a larger impact on the storm's intensity than did the SAL.

In this study, we examine the potential role of the SAL in the case of Hurricane Helene using satellite data, multiple global meteorological analyses, and NAMMA/ SALEX aircraft observations. The system that would become Hurricane Helene emerged from the African coast as a strong tropical wave with a large and welldeveloped circulation late on 11 September 2006. Despite its impressive initial circulation, it intensified slowly (Fig. 1), becoming Tropical Depression (TD) 8 by midday on 12 September, TS Helene by 0000 UTC 14 September, and Hurricane Helene by 1200 UTC 16 September (Franklin and Brown 2008). Subsequently, within $42 \mathrm{~h}$, Helene intensified to a category 3 storm on the SaffirSimpson scale, reaching peak intensity of $\sim 54 \mathrm{~m} \mathrm{~s}^{-1}$ $(955 \mathrm{hPa})$ at 0600 UTC 18 September. Franklin and Brown (2008) attributed the slow initial development prior to 13 September to the presence of easterly shear in the storm environment. Jenkins and Pratt (2008) and Jenkins et al. (2008) suggested that the presence of dust adjacent to the northern side of pre-Helene may have helped to invigorate convection in the system, although the impact of such invigoration on the development of the cyclone remains uncertain. Zipser et al. (2009) suggested that the intensification of Helene may have been delayed by the presence of Saharan air based largely on the assumption that the SAL's influence on intensity is generally negative.

In this study, we focus primarily on the thermodynamic characteristics of the SAL in the vicinity of Helene and their implications for the SAL's impact on 
a) Deep-layer shear

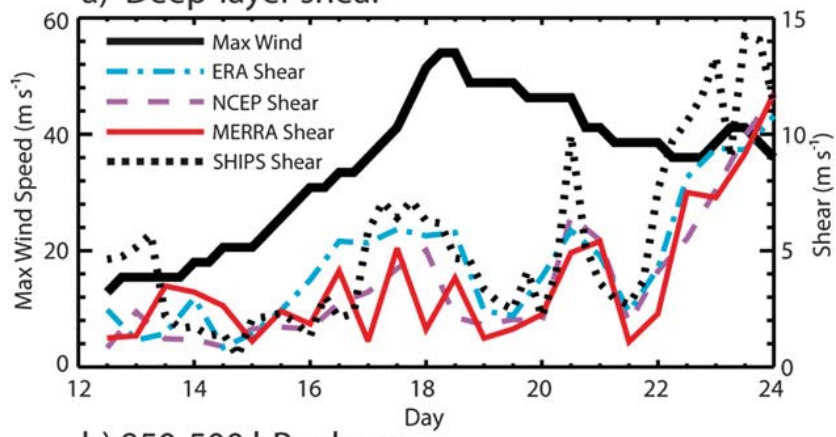

b) $850-500 \mathrm{hPa}$ shear

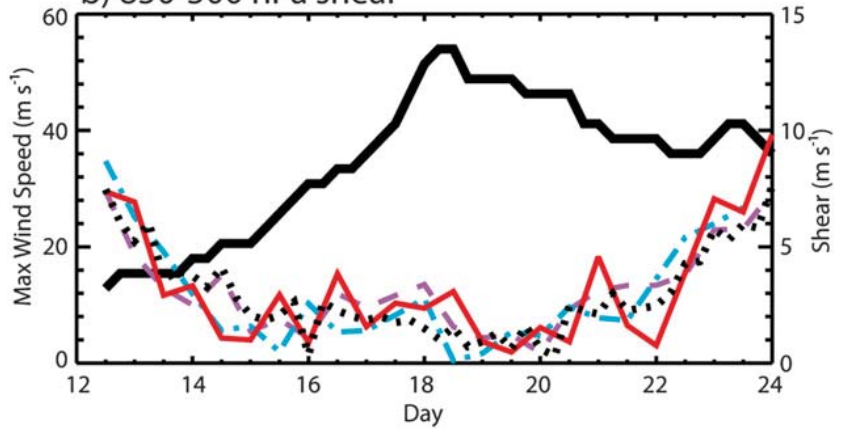

FIG. 1. Time series of estimated maximum surface wind speed for Helene from NHC best track data for 12-24 Sep 2006. Other lines show (a) large-scale deep-layer vertical wind shear (defined in the text) and (b) 850-500-hPa vertical shear estimated from the global analyses and SHIPS as described in section $2 b$.

storm development. We adopt two important working assumptions:

- The "background" environment of the Saharan region (prior to modification by strong surface heating and dry convective mixing) is characterized by deep-layer subsidence and dry air as part of the Hadley circulation (a core reason for the presence of the Saharan Desert). As shown by Braun (2010a), this subsidence is also characteristic of the eastern Atlantic subtropical region and extends well into the central Atlantic.

- The air mass that is truly SAL, as described in the literature (e.g., Carlson and Prospero 1972), has its aforementioned characteristics because of modification of the background air mass by the effects of intense surface heating and deep dry convective mixing over the Sahara. The deep dry convective mixing produces profiles of nearly constant potential temperature and vapor mixing ratio up to the top of the convective boundary layer, which by late afternoon can extend up to $\sim 500 \mathrm{hPa}$ (Messager et al. 2010, see their Fig. 9 for a schematic diagram). This mixing further dries the lower levels of the SAL, but moistens the upper portion of the SAL, sometimes to the point of near saturation (see Fig. 3; also Messager et al. 2010; Ismail et al. 2010; Braun 2010a). Consequently, the characteristic relative humidity (RH) profile of the SAL as it emerges from Africa is that of RH increasing with height [see Figs. 1 and 2 of Karyampudi et al. (1999) for schematic diagrams of SAL structure as it moves over the eastern Atlantic]. The finding of Dunion and Marron (2008) and Dunion (2011) of RH decreasing with height in the SAL over the Caribbean suggests substantial modification of this eastern Atlantic RH profile as the air mass moves westward.

Helene is examined in three stages: pre- and early intensification (from 12 September to mid-15 September), intensification to major hurricane (from mid-15 September to early 18 September), and weakening (early 18 September and onward). We will show that while SAL air was prevalent in the environment of the storm at early stages, it was gradually replaced by a large-scale dry subsiding air mass. While some SAL air was likely drawn into the circulation of Helene during its intensification stage, the evidence suggests that it likely provided only a small braking influence on intensification rather than a substantial suppressing influence. The subsequent weakening of the storm is attributed to an apparent eyewall replacement cycle (ERC) and a marked reduction in SSTs as the storm's forward motion slowed and allowed for greater upwelling of colder ocean waters beneath the storm.

\section{Data}

\section{a. Satellite and field data}

Several NASA satellites currently provide information that is critical to assessing the impacts of the SAL on hurricanes. The Moderate Resolution Imaging Spectroradiometer (MODIS) imagers, on the Terra and Aqua satellites since 2000 and 2002, respectively, provide a measure of the vertically integrated aerosol concentration, or aerosol optical depth (AOD), within the SAL. Here we use the version-5 level- $3^{1}$ gridded products with horizontal grid spacing of $1^{\circ}$ latitude and longitude [available from the Level 1 and Atmosphere Archive and Distribution System (LAADS)]. The Cloud-Aerosol Lidar and Infrared Pathfinder Satellite Observations (CALIPSO) satellite provides accurate estimates of cloud and aerosol layer occurrence, their top and base heights, and optical depths. CALIPSO also provides estimates of aerosol type, including dust, pollution, oceanic

\footnotetext{
${ }^{1}$ Level-1 products are the collected instrument data at full resolution and processed into sensor units. Level-2 products are derived geophysical variables at the same resolution and location as Level 1. Level-3 products consist of gridded variables mapped to uniform space-time grid scales.
} 
aerosols, and continental aerosols (here we use CALIPSO products from version 3.01). The Atmospheric Infrared Sounder (AIRS) and Advanced Microwave Sounding Unit (AMSU) retrieve temperature and humidity profiles that are essential to characterizing the bulk thermodynamic properties of the SAL. Version-5 level-2 orbital products with horizontal resolution of $45 \mathrm{~km}$ and 12 vertical levels between 1000 and $100 \mathrm{hPa}$ are used [available from the Goddard Earth Science Data and Information Services Center (GES-DISC)]. Rainfall information is obtained from multiple products. We use the Tropical Rainfall Measuring Mission (TRMM) multisatellite precipitation product (known as the TRMM 3B42 product, version 6; Huffman et al. 2007) to obtain rain-rate estimates every $3 \mathrm{~h}$ on a grid with $0.25^{\circ}$ horizontal resolution (available from GES-DISC). Rainfall structure information that is coincident in time with the AIRS/AMSU and Aqua MODIS data is obtained from orbital rainfall products (version 6) from the Advanced Microwave Scanning Radiometer (AMSR) for Earth Observing System (AMSR-E) instrument on Aqua (available from the National Snow and Ice Data Center). Sea surface temperatures from TRMM and AMSR-E are obtained from Remote Sensing Systems (version 5, available from www.remss.com).

Of particular note regarding the AIRS/AMSU data is that the temperature data for a particular pressure level is the temperature at that level while the relative humidity for a specified level is the layer-averaged $\mathrm{RH}$ from the specified level to the next level above. For example, AIRS $\mathrm{RH}$ data at $850 \mathrm{hPa}(700 \mathrm{hPa})$ is the average over the layer from 850 to $700 \mathrm{hPa}(700-600 \mathrm{hPa})$. While AIRS clearly lacks the vertical resolution to describe the detailed vertical structure of the SAL, it is capable of resolving the bulk (layer averaged) properties reasonably well (Braun 2010a). Wu (2009) compared soundings from the NAMMA field campaign to AIRS retrieved profiles and found that AIRS relative humidities typically had a small bias $(<7 \%)$ and a root-mean-square error of $\sim 10 \%-15 \%$. For temperature, the bias was $<0.5 \mathrm{~K}$ at most levels, with a root-mean-square error of $\sim 1.2 \mathrm{~K}$.

A NAMMA DC-8 flight occurred on 12 September when Helene first came off of the African coast. Dropsonde data were limited, though, as the DC-8 spent much of the flight obtaining in situ sampling of aerosols at lower levels. Zipser et al. (2009) showed Lidar Atmospheric Sensing Experiment (LASE) data depicting the structure of the dust within part of the SAL near Helene (see their Fig. 12). Here, LASE is used to characterize the coincident dust and moisture data. No additional aircraft observations are available until 15 September. In section $3 \mathrm{a}$, we use soundings from the National Oceanic and Atmospheric Administration (NOAA) G-IV aircraft on 15 September in combination with AIRS data
TABLE 1. Analysis characteristics for NCEP, ERA-Interim, and MERRA. All analyses are available every $6 \mathrm{~h}$. Under vertical pressure levels, the number in parentheses is the number of levels below and including $100 \mathrm{hPa}$.

\begin{tabular}{lcc}
\hline \hline Analysis & Horizontal resolution & $\begin{array}{c}\text { Vertical } \\
\text { pressure } \\
\text { levels }\end{array}$ \\
\hline $\begin{array}{l}\text { NCEP GDAS } \\
\text { Final Analyses }\end{array}$ & $1^{\circ}$ & $26(21)$ \\
ERA-Interim & $0.7^{\circ}$ & $37(27)$ \\
MERRA & $0.5^{\circ} \times 0.66^{\circ}$ for horizontal winds, & $42(25)$ \\
& $\begin{array}{l}\text { temperature, water vapor; } \\
1.25^{\circ} \text { for vertical motion }\end{array}$ & \\
\hline
\end{tabular}

to describe the vertical structure of the dry air mass that wrapped around Helene during its intensification stage.

\section{b. Global meteorological analyses}

To complement the satellite and aircraft data, three different global meteorological analyses are used to investigate the evolution of Helene's environment: NCEP's GDAS final analyses, the European Centre for Medium-Range Weather Forecasts (ECMWF) Interim Re-Analysis (ERA-Interim), and NASA's Modern-Era Retrospective Analysis for Research and Applications (MERRA). Characteristics of the analyses are provided in Table 1 . The analyses use different global models and analysis systems and have different resolutions. Neither the NCEP nor ERA analyses account for the radiative effects of dust, while MERRA uses climatological dust distributions to account for dust radiative forcing, but cannot account for the variability of dust forcing associated with individual dust outbreaks. As a result, on the scale of an individual dust outbreak, the analyses may underestimate the inversion strength at the base of the SAL (DV04) and may not adequately capture the influence of the dust on vertical motions (Karyampudi and Carlson 1988). Close comparison of the analyses over the period of 12-18 September suggests that they provide very similar depictions of the SAL and the evolving environment of Helene.

The global analyses are also used to estimate the large-scale vertical wind shear during the period of 1223 September. Two estimates of vertical shear are calculated. A deep-layer shear value is calculated as the difference between the mean winds, averaged over a $10^{\circ}$ latitude and longitude box centered on the storm, in the 300-200- and 900-700-hPa layers. These layers are similar to those used by DV04. A low-level shear value is obtained over a similar averaging area, but taking the difference in horizontal mean winds at 850 and $500 \mathrm{hPa}$ in order to assess shear associated with the AEJ. Both shear estimates are also compared to values from the 
Statistical Hurricane Intensity Prediction System (SHIPS) model (DeMaria and Kaplan 1994, 1999) for the 850-200and $850-500-\mathrm{hPa}$ layers. SHIPS shear parameters are averaged over radii from 200 to $800 \mathrm{~km}$. Time series of the mean shear for each analysis are shown in Fig. 1.

\section{c. Identifying $S A L$ air}

Identifying the boundaries of SAL air masses, particularly from satellite data, is a major challenge. MODIS AOD, where available, provides a clear indication of regions with dust within the atmospheric column, but not the altitudes at which dust is present. A small AOD value may indicate a deep, but diffuse layer of dust or a shallow and concentrated layer. Although any threshold is arbitrary, in this study, we choose an AOD threshold of 0.3 to approximate the boundary of dust layers likely to extend through the typical SAL levels from $\sim 850$ to $600 \mathrm{hPa}$ or higher. Inspection of CALIPSO data in the regions around Helene suggests that $A O D$ values below this threshold usually corresponded to shallow layers of aerosols confined mostly to the boundary layer.

With the AIRS data, our intent is to identify sources of dry air and their possible intrusion into the storm circulation. The SAL tends to be driest in its lower levels such that the lowest humidities will generally be best observed in the AIRS-retrieved humidity fields in the 850-700-hPa layer. Where MODIS indicates dusty air, a relative humidity threshold of $60 \%$ in this layer is used to approximate the boundary between dry SAL air and more humid air masses. While this threshold is an inexact boundary of the SAL, it is a reasonable means of identifying the locations of the dry air.

A primary application of the NCEP and ERA-Interim analyses is the calculation of 6-day backward trajectories to determine the origins of air masses in the tropical cyclone environment. ${ }^{2}$ Trajectories are calculated using the Integrated Data Viewer (IDV) employing a RungaKutta forward integration and using linear interpolation in time to advance trajectories with a 1-h time step. Backward trajectories are seeded at every grid point within specified domains at multiple levels (850, 700, 600 , and $500 \mathrm{hPa}$ ). For each trajectory, the originating location of the trajectories (i.e., the computed position 6 days earlier) is identified and plotted at the location of the trajectory seed, allowing maps to be drawn of originating latitude and longitude and total pressure-altitude

\footnotetext{
${ }^{2}$ We do not use MERRA for trajectory calculations because vertical motion fields are only provided on a coarser horizontalresolution grid compared to available higher-resolution horizontal wind fields.
}

change along the trajectory. In addition, the trajectories are used to identify air parcels of likely SAL origin and non-SAL origin. Air parcels are identified as likely SAL if they pass over northern Africa between $14^{\circ}$ and $36^{\circ} \mathrm{N}$ and below $575 \mathrm{hPa}$ (the observed top of the SAL in this case). Parcels passing over the same region, but above $575 \mathrm{hPa}$, are identified as possible SAL, although their higher altitude suggests that their origin is actually above the SAL. All others are considered non-SAL.

\section{Pre- and early intensification stages}

\section{a. SAL and non-SAL airmass evolution in relation to Helene}

The relationship between the evolution of Helene and the SAL dust is indicated in Fig. 2, which shows daily MODIS AOD and TRMM 24-h rainfall accumulation for 4 days between 11 and 17 September. Note that areas of missing MODIS data are indicated by gray shading. Late on 11 September (Fig. 2a), the mesoscale convective system that became Helene moved off the African coast just southward of a region of enhanced AOD values between $15^{\circ}$ and $23^{\circ} \mathrm{W}$. Two days later (Fig. 2b), a broad region of dust extended well westward of TD 8, with the highest dust concentrations along the northwestern edge of the depression. A narrow tail of lower AOD values extended toward the African coast to the north of the system. By 15 September (Fig. 2c), the dust region had largely moved off to the west well ahead of Helene (now a tropical storm). A tongue of low AOD $(<0.3)$ air separated Helene from the dust layer although a narrow band of moderate AOD values $(>0.3)$ still extended into the southern part of the storm. By 17 September (Fig. 2d), the environment around Helene was largely dust free (although data are missing very close to the storm). These results suggest that the primary time of potential influence of Saharan dust was prior to 15 September.

The thermodynamic structure of the SAL on 12 September is illustrated by a pair of dropsonde soundings (Fig. 3) from the NASA DC8 taken within the dust layer on the northwestern side of the storm (see Figs. 4 and 13a for the locations). As seen in the northern sounding closest to the dust AOD maximum (at 1221 UTC, blue lines in Fig. 3a), the vertical structure exhibited a cool and moist boundary layer capped by a strong inversion near $900 \mathrm{hPa}$. Between 800 and $600 \mathrm{hPa}$, the temperature in the SAL was nearly isentropic and the vapor mixing ratio was nearly constant at $6 \mathrm{~g} \mathrm{~kg}^{-1}$. The resultant $\mathrm{RH}$ field therefore increased from about $28 \%$ near the base of the SAL to $90 \%$ near its top. This profile is consistent with those discussed in Carlson and Prospero (1972), Karyampudi and Carlson (1988), Ismail et al. (2010), Messager et al. (2010), and Braun (2010a). 

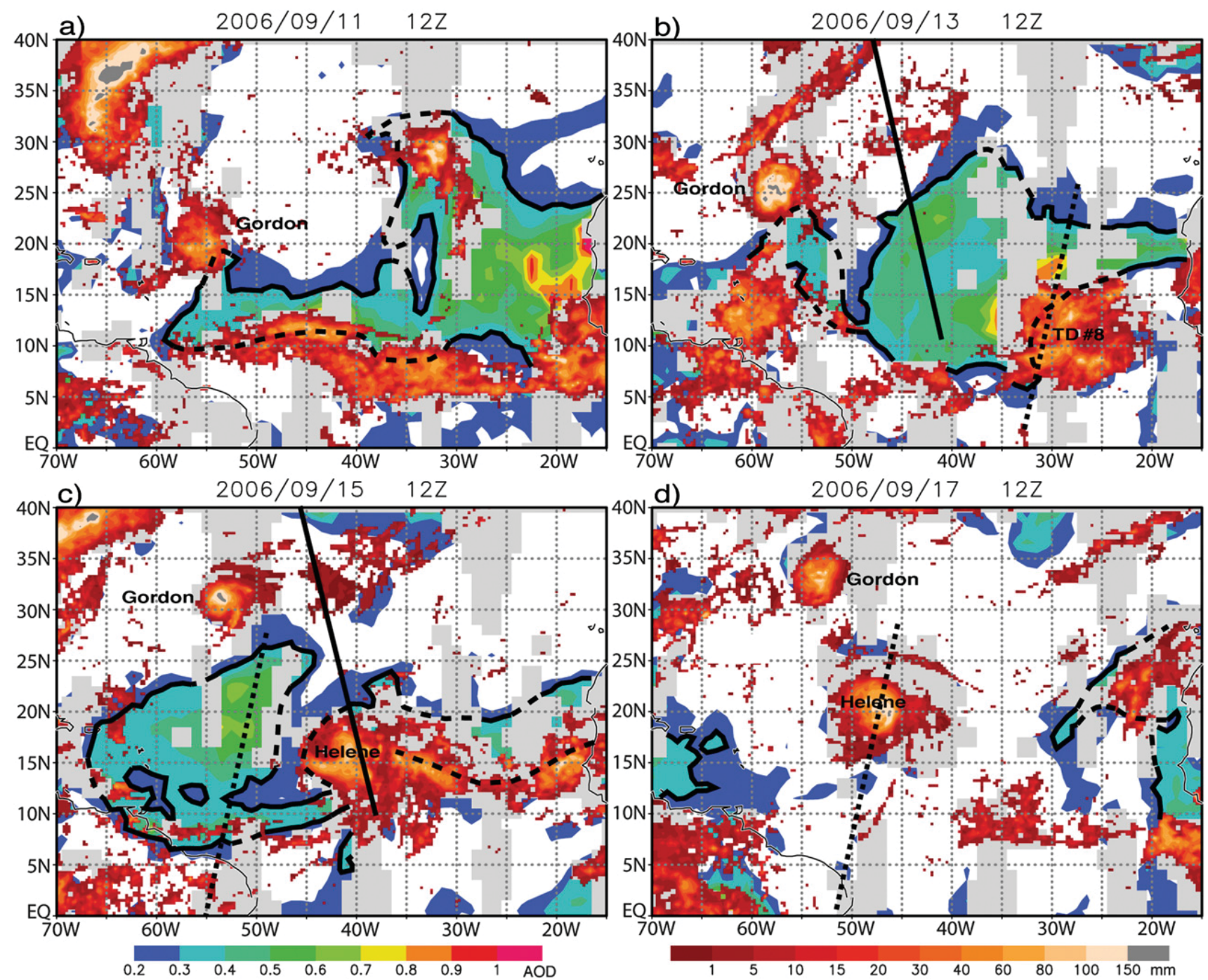

FIG. 2. (a)-(d) MODIS AOD (left color scale) and TRMM 3B42 24-h accumulated rainfall (right color scale) for the indicated dates. White shading indicates AOD values $<0.2$, gray shading indicates missing data. AOD values valid for the time period between 1030 and 1330 local standard time (LST) while the rainfall data are accumulated from 0000 UTC of the given day to 0000 UTC of the next day. Black curves indicate approximate MODIS dust boundaries (AOD >0.3), dashed where approximated in areas of missing data. Straight black lines indicate CALIPSO overpasses, solid for daytime overpasses (coincident with the MODIS AOD data) and dashed for nighttime overpasses that are offset by $12 \mathrm{~h}$ from the data shown in the figure. The positions of Gordon, TD 8, and Helene are indicated.

The second sounding (red lines) obtained just west of pre-Helene at 1242 UTC shows a dramatically modified set of profiles. The boundary layer depth had nearly doubled and the air within the SAL was cooler and more moist relative to the first sounding. The minimum relative humidity was $54 \%$ near $780 \mathrm{hPa}$ compared to the much lower values (28\%) farther northward. The LASE specific humidity and aerosol scattering ratio (ASR) data corresponding to the flight leg in Fig. 12 of Zipser et al. (2009) are also shown in Fig. 3. The dust layer (Fig. 3c) extended up to about $4.6 \mathrm{~km}$ along most of the flight leg, but with its top dropping downward to about $3 \mathrm{~km}$ near the southern edge of the flight leg closest to the storm. According to the specific humidity and ASR data, the boundary layer deepened to the south, with the heavy dust in the boundary layer coinciding with very moist conditions (RH $\sim 90 \%$ according to the 1242 UTC sounding). Furthermore, moisture (Fig. 3b) above the boundary layer and up to $4 \mathrm{~km}$ in height gradually increased, with $700-\mathrm{hPa}$ vapor mixing ratios $\left(6-9 \mathrm{~g} \mathrm{~kg}^{-1}\right)$ approaching typical nonSAL tropical values [see Fig. 7 of Dunion and Marron (2008); Fig. 6 of Dunion (2011)].

The RH and temperature structure from AIRS/AMSU observations in the environment of the early disturbance at $\sim 0300$ UTC 12 September are shown in Figs. 4 and 5, respectively. Overlaid in the data void regions of the AIRS plots are the coincident AMSR-E derived instantaneous rainfall rates. At this time, the precipitation 

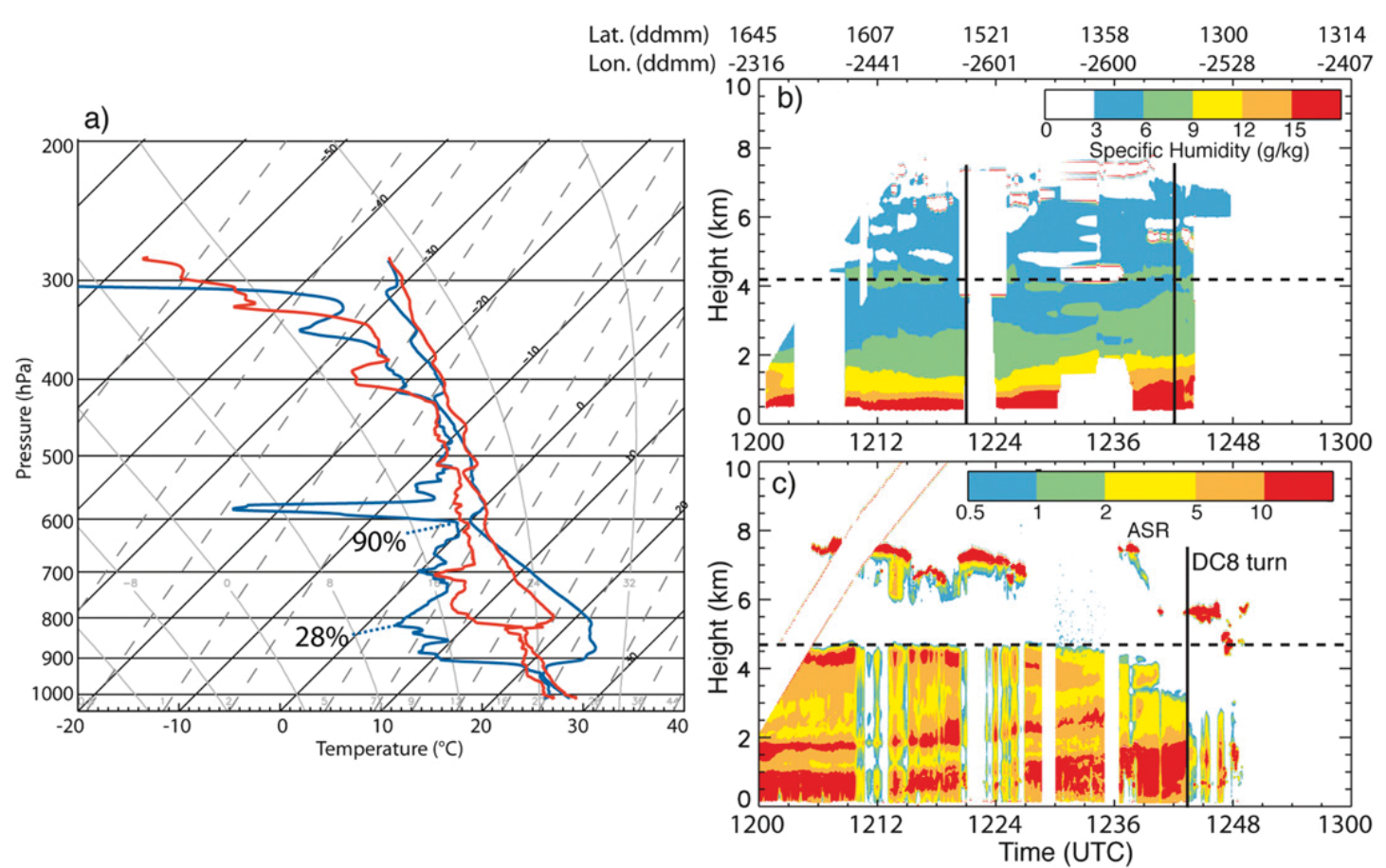

FIG. 3. (a) Skew $T-\log p$ diagrams for two dropsondes from the NASA DC8 aircraft. Blue lines show the drop at 1221 UTC and red lines at 1242 UTC 12 Sep. (b) LASE water vapor specific humidity and (c) aerosol scattering ratio between 1200 and 1300 UTC 12 Sep. Latitude and longitude positions (in degrees and minutes) for the DC 8 are provided along the top of (b). The vertical lines in (b) indicate the times of the two dropsondes while the vertical line in (c) indicates the time of the turn by the DC8 from a southerly heading to an easterly heading. The position of the flight segment is indicated in Fig. $4 \mathrm{a}$. The dashed line at $\sim 4.7 \mathrm{~km}$ indicates the approximate top of the SAL.

structure consisted of an east-west-oriented band of precipitation along $\sim 7^{\circ} \mathrm{N}$ and two north-south-oriented bands near $21^{\circ}$ and $18.5^{\circ} \mathrm{W}$. These precipitation features were embedded within a moist region at all levels up to $500 \mathrm{hPa}$ except for the western portion of the east-westoriented band that appeared to be adjacent to or even topped by dry air above $500 \mathrm{hPa}$ (Figs. 4d).

Dry SAL air (RH $<\sim 60 \%$ ) was present in the 850 700-hPa layer (Fig. 4a) to the north and west of the north-south-oriented precipitation bands. Consistent with the NAMMA soundings in Fig. 3, which indicated a moist top of the SAL near $600 \mathrm{hPa}$, the $700-600-\mathrm{hPa}$ RH (Fig. 4b) in the SAL air was more moist with values of $50 \%-70 \%$ over most of the region north of the storm except for a narrow region immediately north of the moist area containing the tropical disturbance. Dry air surrounded the northern and western sides of the disturbance well above the SAL in the 500-400-hPa layer (Fig. 4d). The low humidities in the same region in the 600 500-hPa layer (Fig. 4c) were likely the result of subsidence of dry air from above the SAL rather than from the SAL itself given the observed SAL top at $575 \mathrm{hPa}$ and the higher humidity values in the layer below (Fig. 4b).

Warm temperatures associated with the SAL at low levels (Fig. 5) were well observed by AIRS and acted to suppress convection to the north of the AEJ axis, while the convective rainbands were located in the cooler air to the south of the jet. Isotachs at $700 \mathrm{hPa}$ from the NCEP (Fig. 5a) and ERA-Interim (Fig. 5b) analyses at 0000 UTC 12 September show that the axis of the AEJ was north of the convection along a zone of strong 850- and 700-hPa temperature gradient. Braun (2010a) argued that suppression of convection by the strong temperature inversion at the base of the SAL to the north of the AEJ axis is not detrimental to development of the vortex since convection, were it to occur so far from the vortex center, would be unlikely to contribute to vortex spinup. South of the jet axis, where the strong SAL inversion is generally not present, convection is able to occur in the region of enhanced background cyclonic vorticity associated with the cyclonic shear side of the jet. This juxtaposition of convection and vorticity may be important for spinup of the vortex (Ross et al. 2009; Berry and Thorncroft 2012).

At 0643 UTC 12 September, QuikSCAT measured the surface wind field shortly after pre-Helene had emerged off of Africa (Fig. 6a). Erroneously high wind speeds are known to occur in areas of precipitation (Portabella and Stoffelen 2001; Chelton et al. 2006; Brennan et al. 2009), and these regions provide an indication of the qualitative rainfall 

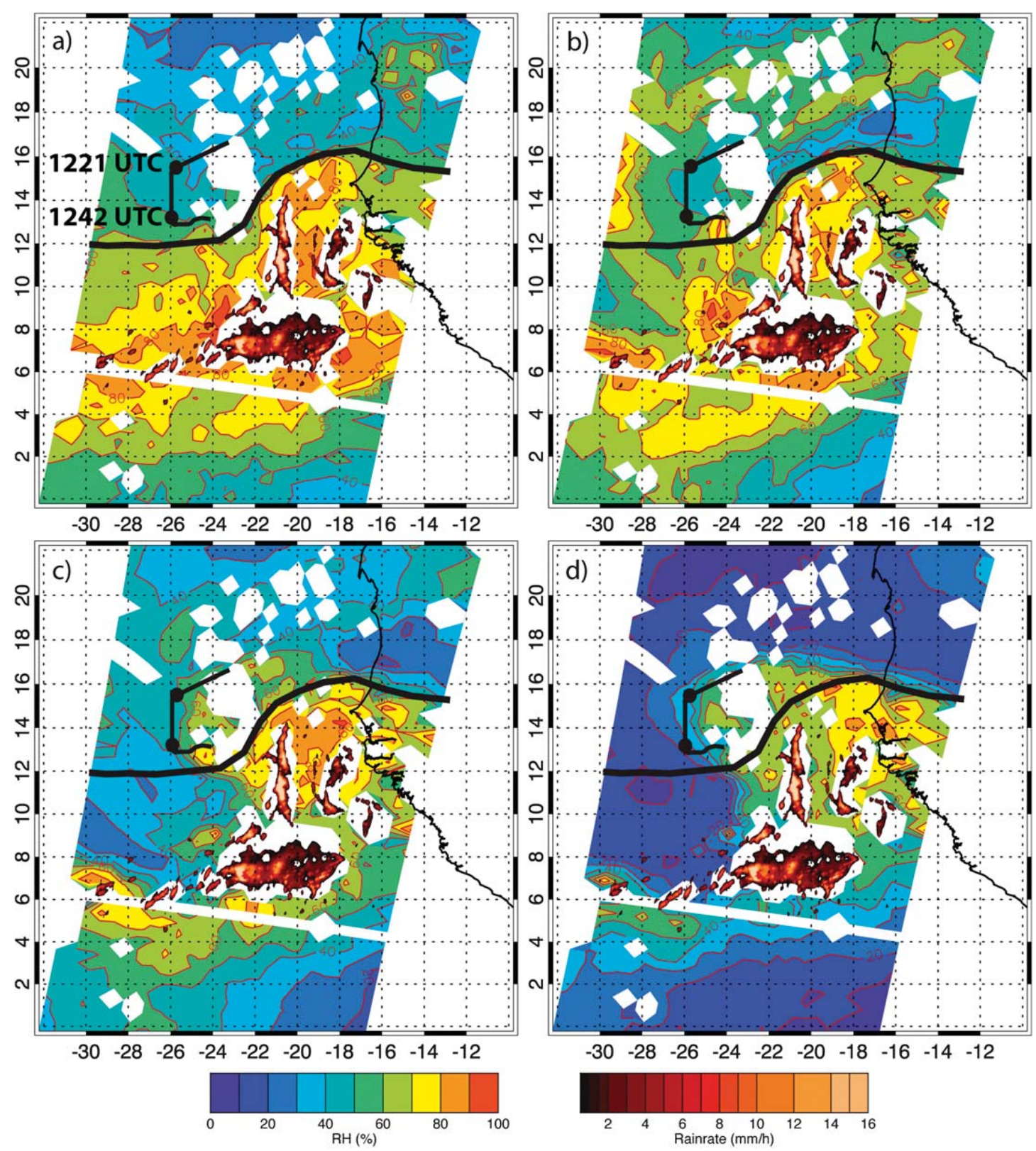

FIG. 4. AIRS/AMSU layer-averaged RH (left color scale) for $\sim 0300$ UTC 12 Sep for the (a) 850-700-, (b) 700600-, (c) 600-500-, and (d) 500-400-hPa layers. Superimposed within AIRS data gaps are the coincident AMSR-E derived surface rainfall estimates (right color scale). The black solid curve, following closely the $60 \% \mathrm{RH}$ contour in the 850-700-hPa layer, shows the approximate southern boundary of the dry SAL air. The short black line segment and two circles show the locations of the LASE data and two dropsondes in Fig. 3 approximately $9.5 \mathrm{~h}$ after the time of the AIRS data. Accounting for an estimated westward storm motion of $7 \mathrm{~m} \mathrm{~s}^{-1}$, the dropsonde locations were about $2.2^{\circ}$ closer to the convective line at the time of the AIRS overpass.

structure at the time. The high-wind data and the rain probability contour show the location of a northwestsoutheast-oriented rainband that likely corresponded to one of the north-south-oriented bands in Figs. 4 and 5. The surface flow was characterized by large-scale cyclonic curvature, with one area of weak surface winds located north of the $\mathrm{AEJ}\left(>16^{\circ} \mathrm{N}\right)$ and another immediately on the eastern side of the rainband at the latitude $\left(\sim 12^{\circ} \mathrm{N}\right)$ of the storm center 5 hours later when the National Hurricane Center first identified TD 8. Strong surface inflow into the convective band occurred on the band's western side. By 1830 UTC (Fig. 6b), a clearly defined center location was found well to the east of the leading (most western) rainband and just north of a southwest-northeast-oriented band. The 


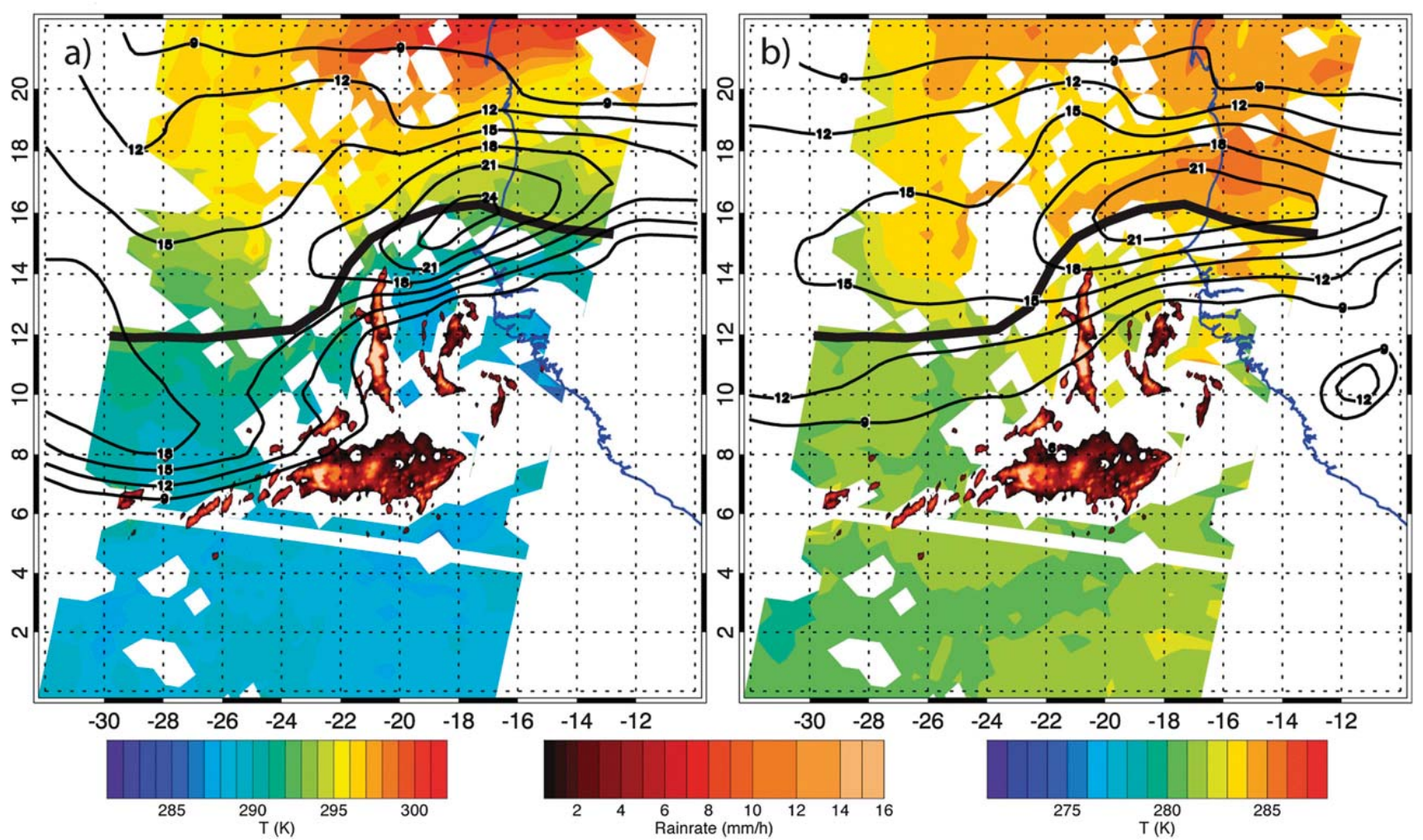

FIG. 5. AIRS/AMSU temperature for $\sim 0300$ UTC 12 Sep for (a) 850 (left color scale) and (b) $700 \mathrm{hPa}$ (right color scale). Superimposed within AIRS data gaps are the coincident AMSR-E derived surface rainfall estimates (middle color scale). The thick black solid curve shows the approximate southern boundary of the dry SAL air in the 850-700-hPa layer from Fig. 4. Black contour lines show isotachs at $700 \mathrm{hPa}$ from the (a) NCEP and (b) ERA-Interim analyses at increments of $3 \mathrm{~m} \mathrm{~s}^{-1}$ starting at $9 \mathrm{~m} \mathrm{~s}^{-1}$.

circulation was quite large at this time with an estimated radius of maximum wind on the order of $\sim 200 \mathrm{~km}^{3}{ }^{3}$ The southern boundary of the SAL (inferred from the humidity and precipitation fields in Figs. 4 and 8) suggests that the dry SAL air was located just north and west of the precipitation indicating some potential for dry air to interact with the convection on the western edge of the storm. However, the dry air was approximately $400 \mathrm{~km}(300 \mathrm{~km})$ to the west (north) of the storm center at 1830 UTC.

Deep-layer shear (Fig. 1a) was generally weak through the early stages of Helene (12-15 September) although SHIPS suggested a higher value early on. In contrast, the 850-500-hPa shear (Fig. 1b) was from the east at $7-9 \mathrm{~m} \mathrm{~s}^{-1}$ on 12 September, but weakened to $3 \mathrm{~m} \mathrm{~s}^{-1}$ by 14 September. The initially strong low-level easterly shear could have contributed to the linear organization of the convection on 12 September (i.e., the horizontal vorticity associated with the easterly vertical shear was likely balanced by that associated with the cold pool; Rotunno et al. 1988). As suggested by DV04 and Vizy and Cook (2009), the

\footnotetext{
${ }^{3}$ An exact radius is difficult to determine given the wind speed bias in the precipitation regions.
}

vertical shear might also contribute to a tilt of the vortex. The global analyses consistently show a northward tilt of the vortex (i.e., to the right of the easterly shear vector) in the layer between 850 and $500 \mathrm{hPa}$ (see the example from NCEP analyses in Fig. 7), although they vary in the direction of the tilt in the zonal direction [(little zonal tilt in NCEP, little tilt or an eastward tilt in ERA, and a westward tilt in MERRA) (the latter two not shown)]. The NCEP analyses show that from 1200 UTC 12 September to 0000 UTC 14 September (Figs. 7a-d) the midlevel vorticity maximum was located in the region of strong cyclonic shear on the southern side of the AEJ wind maximum, with the $850-\mathrm{hPa}$ vorticity maximum located farther to the south. During 14 September (Figs. 7e,f), the vortices became vertically aligned as the strength of the AEJ (and the low-level easterly shear) decreased. Note that the strengthening of Helene on 14 September did not occur as the AEJ moved away from the storm, but instead occurred as the jet weakened on the northwestern side of it. This result is consistent with Ross et al. (2009) who argued that the location of the strongest AEJ winds on the northwestern side of the disturbance during 10-14 September contributed to barotropic energy growth during Helene's TD stage. 
a) 0643 UTC September 12

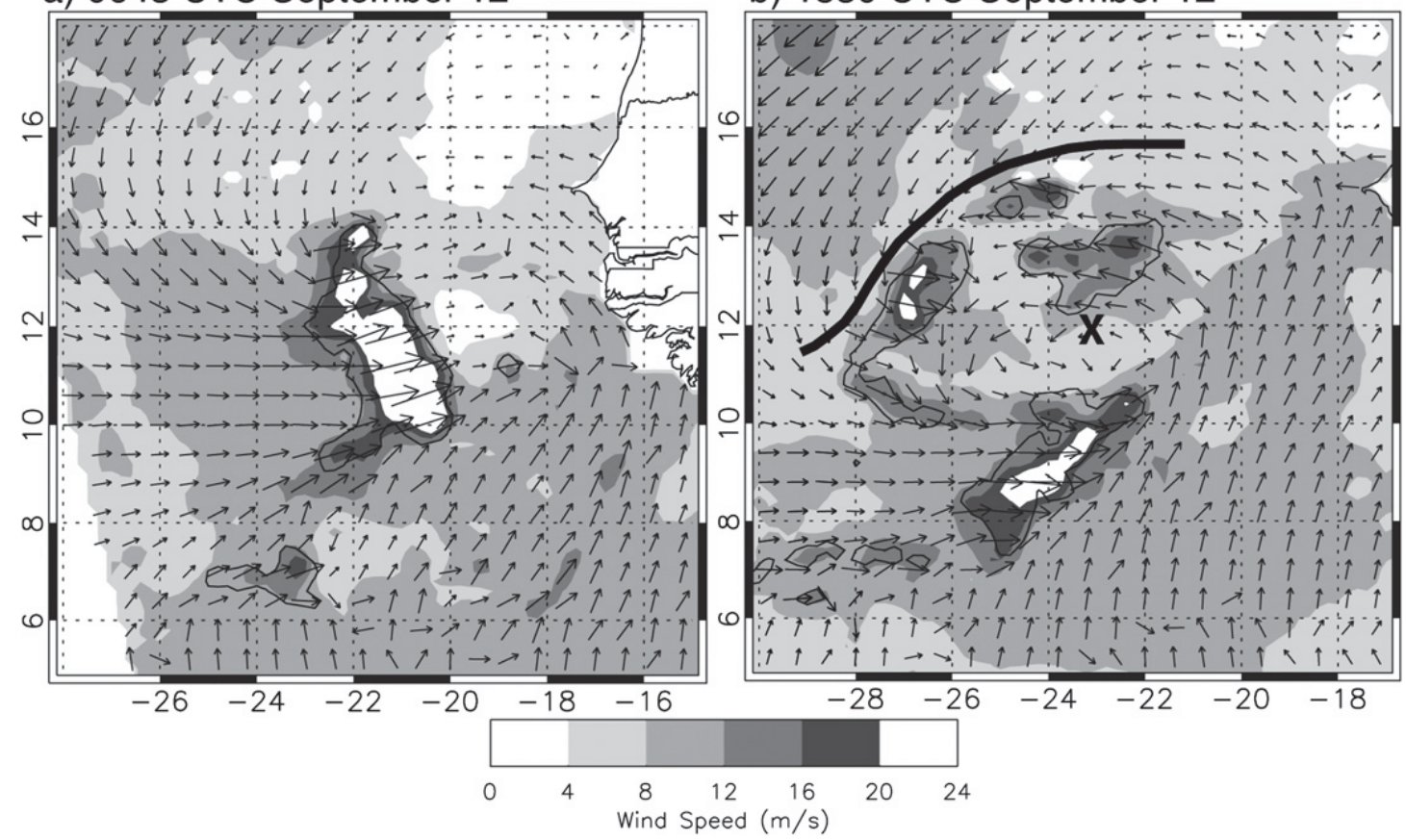

FIG. 6. QuikSCAT surface wind speeds (shading) and vectors for (a) 0643 and (b) 1830 UTC 12 Sep. Thin solid contour lines enclose areas with rainfall probabilities exceeding $50 \%$. In (b), the estimated center of TD 8 from the $\mathrm{NHC}$ is indicated by the $\times$. The bold curve indicates the approximate southern boundary of the dry midlevel SAL air based upon the data in Fig. 4a.
Under conditions of easterly $850-500-\mathrm{hPa}$ vertical wind shear, low-level inflow would likely have occurred on the western side of the storm and may have brought SAL air within range to be ingested into the rainband there (Fig. 6b). Unfortunately, information from the 12 September NAMMA DC-8 flight and from satellite data are insufficient to determine whether pronounced cold pool formation occurred and the extent to which such a cold pool might have impacted the energy available to the developing vortex. This topic will need to be addressed in a future study using a well-constructed numerical simulation.

Relative humidity (Fig. 8) and temperature (not shown) fields from AIRS at $\sim 0400$ UTC 13 September are comparable to those observed a day earlier. As seen in AMSR-E data, precipitation bands exhibited greater cyclonic curvature on the western side of TD 8 (centered at $\sim 11.9^{\circ} \mathrm{N}$, $25^{\circ} \mathrm{W}$ ). The SAL dry-air boundary in the $850-700-\mathrm{hPa}$ layer (Fig. 8a) was located $\sim 350 \mathrm{~km}$ to the north and $\sim 500 \mathrm{~km}$ to the west of the center and suggests that, despite the low-level vertical wind shear, there was little intrusion of the dry air into the vortex. Relative humidities in the 700-600- and 600-500-hPa layers to the north of the storm decreased by $10 \%-20 \%$ compared to a day earlier while conditions in the 500-400-hPa layer remained quite dry.
A CALIPSO overpass (Fig. 9a), coincident in time with the AIRS data (location given by the dotted line in Fig. 8 ) and $\sim 600 \mathrm{~km}$ to the west of the center of TD 8 , measured the vertical structure of the dust layer in that area. To the northwest of the storm (right side of the image), the deep dust layer extended from the boundary layer to $\sim 4.5 \mathrm{~km}$ adjacent to the western side of the storm, but decreased in height farther northward to about $4 \mathrm{~km}$ near $24^{\circ} \mathrm{N}$ and $2.5 \mathrm{~km}$ farther northward. On the left side of the image in Fig. 9a, an elevated dust layer was present between $\sim 3$ and $4-\mathrm{km}$ altitude and extended to lower altitudes closer to the cloud system, indicating that some dust had made its way around to the southwestern edge of TD 8. According to AIRS data within this dust layer to the southwest and west of TD 8, conditions were relatively moist in the 850-700hPa layer (Fig. 8a) and there was a narrow tongue of dry air in the 700-600-hPa layer (Fig. 8b). The dry tongue was also seen, and was more prominent, at higher levels above the dust (Figs. 8c,d), suggesting that a major portion of the dry-air tongue between 700 and $600 \mathrm{hPa}$ was a result of downward advection of dry air from above the SAL. However, some dry SAL air was likely present at lower levels but unresolved by the AIRS profiles. Farther westward, CALIPSO (Fig. 9b) found a deep, but elevated dust layer associated with 
a) 12 UTC 12 Sept

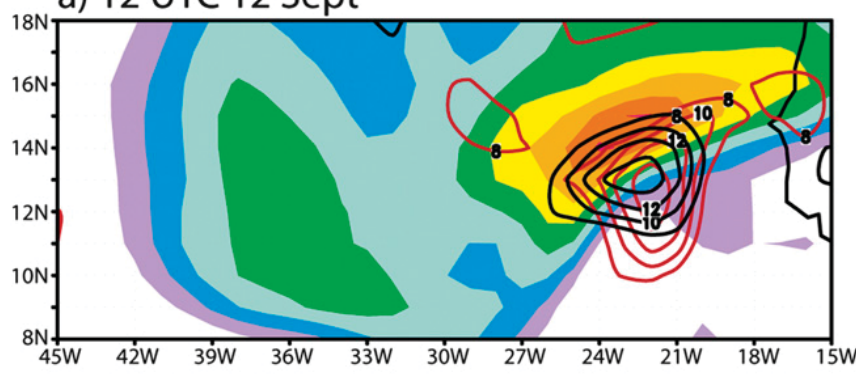

C) 12 UTC $13 \mathrm{Sept}$

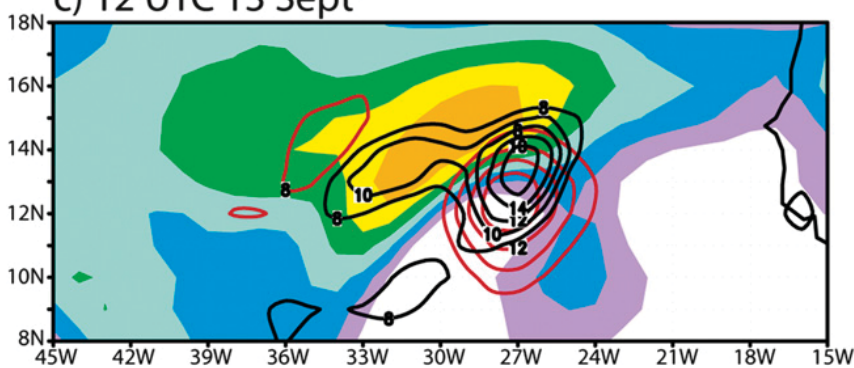

e) 12 UTC 14 Sept

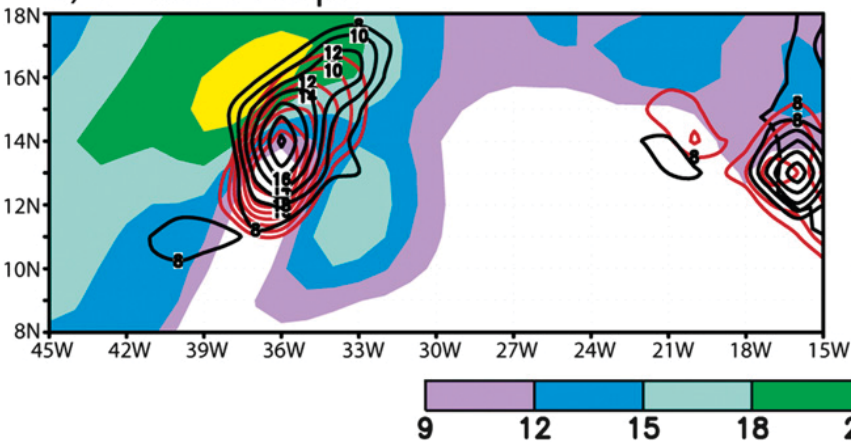

b) 00 UTC $13 \mathrm{Sept}$

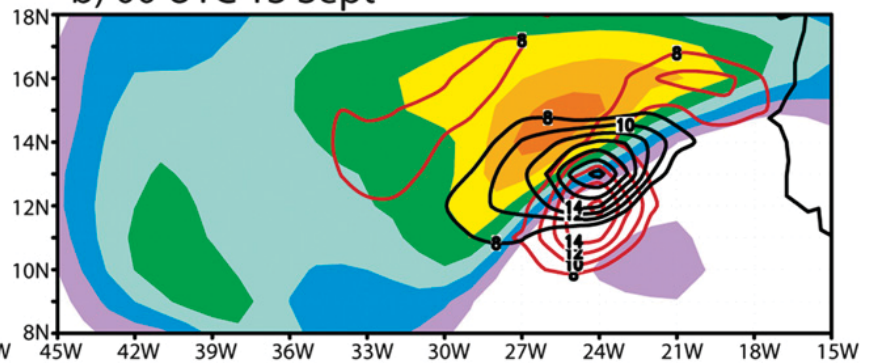

d) 00 UTC 14 Sept

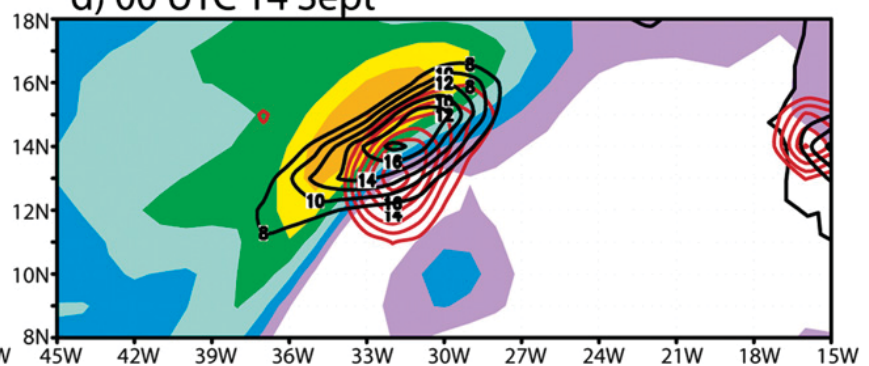

f) 00 UTC $15 \mathrm{Sept}$

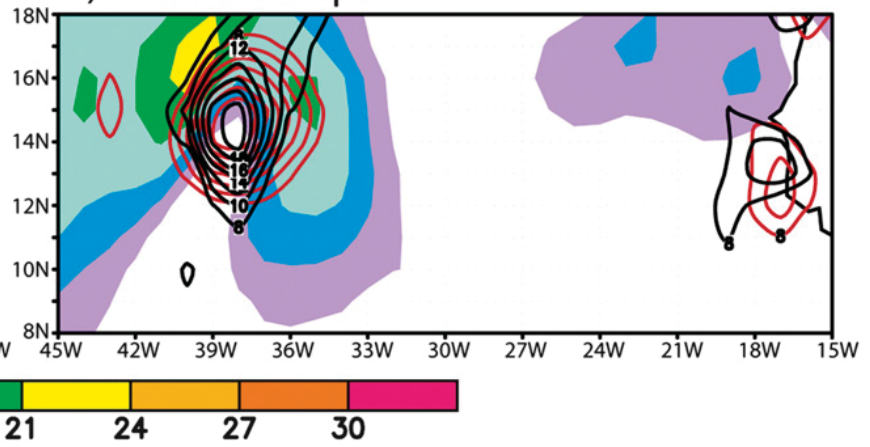

FIG. 7. NCEP analyses of the evolution of the 700-hPa AEJ wind speed (shading) and 500- (black) and 850-hPa (dark red) relative vorticity at the indicated times. Vorticity contours are drawn at $2 \times 10^{-5} \mathrm{~s}^{-1}$ intervals between 8 and $20 \times 10^{-5} \mathrm{~s}^{-1}$.

the broad area of SAL air moving westward well ahead of the storm (solid line in Fig. 2b).

The AIRS overpass at 0300 UTC 14 September missed most of the storm while the $\sim 1500$ UTC overpass provided coverage of the storm, but missed the region of dry air to its west. Consequently, Fig. 10 shows a merged analysis of data from the two times, valid at 1500 UTC, with the 0300 UTC data (gray shading) shifted westnorthwestward to account for storm motion between the two times. The most notable feature was the pronounced drying of the air at most levels since the time shown in Fig. 8. Whereas on 13 September relative humidities in the 700-600-hPa layer were generally $>30 \%-40 \%$, by 14 September large areas with RH of $10 \%-30 \%$ were observed to the north and west of now TS Helene. Similar extensive drying was also seen in the 600-500-hPa layer and less so in the 850-700-hPa layer. A very sharp boundary between the moist air in TS Helene and the dry air to the north occurred at all levels including those above the earlier-observed SAL top $(600 \mathrm{hPa})$, with this dry-air boundary generally about $350-400 \mathrm{~km}$ north of the storm center. To the south of the center (between 200 and $400 \mathrm{~km}$ ), humidities in the 850 700-hPa layer remained high while at higher levels the air became increasingly drier with height. To the extent that dry air was impacting storm development, it would most likely have originated with this dry air intrusion most prominently seen above $700 \mathrm{hPa}$ to the south of the storm.

By $\sim 1600$ UTC 15 September (Fig. 11), dry air extended cyclonically from the northern side of Helene around to the southern and possibly eastern sides. To the north of the storm, CALIPSO data (Fig. 9c) showed very little dust in the dry air. All of the aerosol were contained in the layer below $2 \mathrm{~km}$ and consisted of a mix of aerosol types including dust, marine aerosols, and some polluted continental air. Well to the west (Fig. 9d, see location in Figs. 2c and 11), a more prominent aerosol 

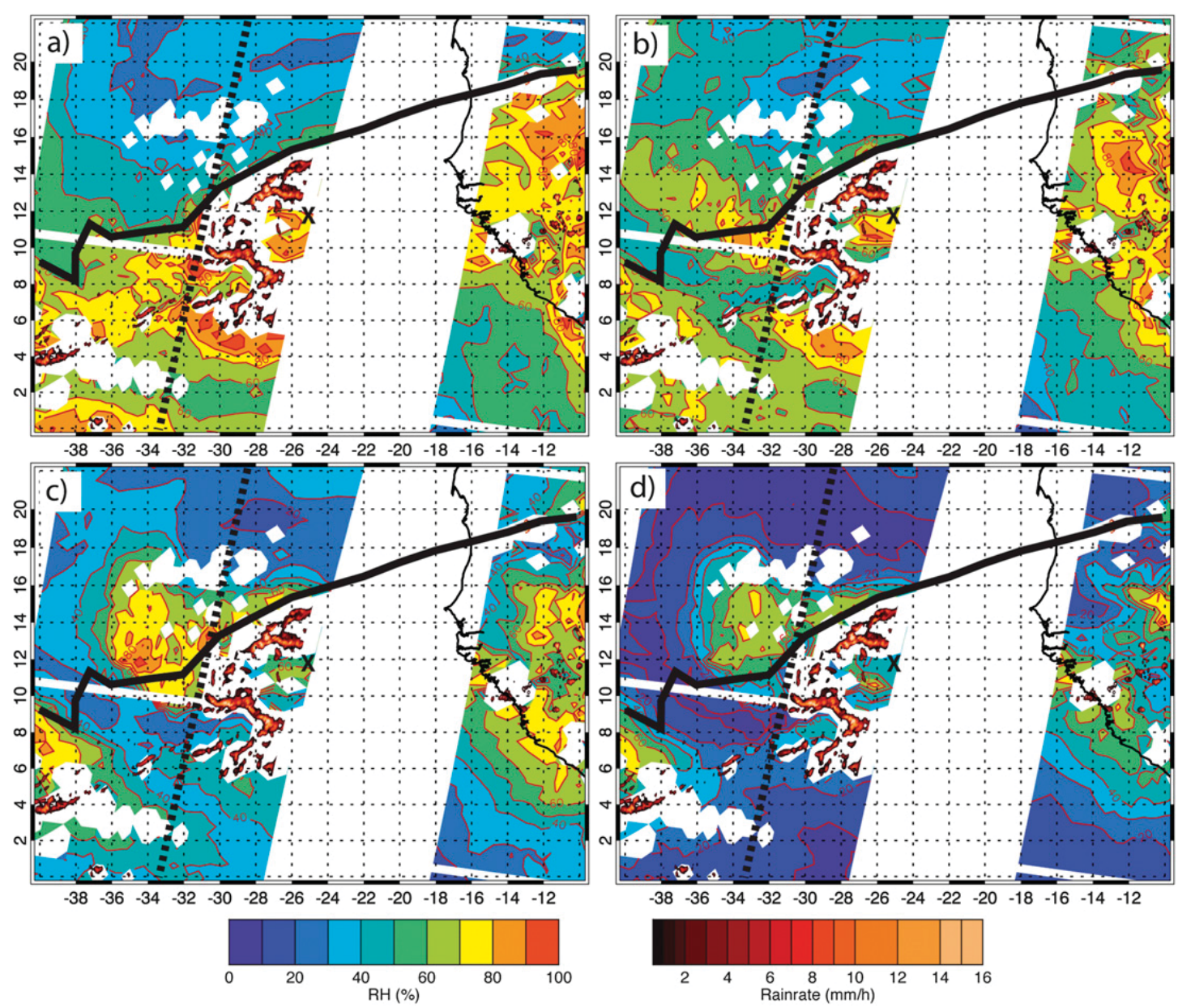

FIG. 8. As in Fig. 4, but for $\sim 0400$ UTC 13 Sep. The black dotted line shows the position of the coincident CALIPSO overpass in Fig. 9a. The estimated center of Helene from the NHC is indicated by the $\times$.

layer was contained in the westward moving SAL air, but did not interact with the storm in any direct way. As at earlier times, the RH generally decreased with increasing height (not shown), including along the southern side of the storm where the dry air got closest to the precipitation. The major precipitation band was on the southern side of the storm, separating the storm inner-core region from the nearby dry air. Although few AIRS pixels were available near the rainband on the southeastern side of the storm, the figure suggests a pattern in which dry air may have been wrapping around to the eastern side. ${ }^{4}$

To this point, AIRS retrievals have been used without direct validation of their accuracy in the tropical storm environment and with limited information within the cloud system. The NOAA G-IV began surveying Helene

\footnotetext{
${ }^{4}$ Pixels near cloud boundaries may exhibit a dry bias due to clouds (B. Lambrigtsen and S. Wong, 2011, personal communication) and should be viewed with some caution.
}

daily beginning 15 September and dropsonde locations are indicated in Fig. 11 for this date. Figure 12 shows temperature, relative humidity, and wind speed profiles for 15 of the dropsondes on 15 September (positions indicated by filled circles in Fig. 11). Included within this set of profiles are data from dropsondes in the very dry air to the west of Helene as well as dropsondes along the dry intrusion on the southern and eastern sides of the storm. Where available, nearly coincident-in-time AIRS retrievals of RH are indicated by the black symbols, showing good agreement between the AIRS and dropsonde profiles in most cases. Note, however, that AIRS retrievals are layer-averaged values and cannot resolve the high vertical resolution variations seen in the dropsonde data. The largest disagreements (S10-S12) are associated with AIRS pixels that have quality indicators suggesting possible cloud contamination and the potential for a dry bias at midlevels. At the location of sounding S7, the AIRS RH profile also shows departures from the dropsonde data. This AIRS profile is not cloud contaminated, but there is an almost 6-h time difference between 

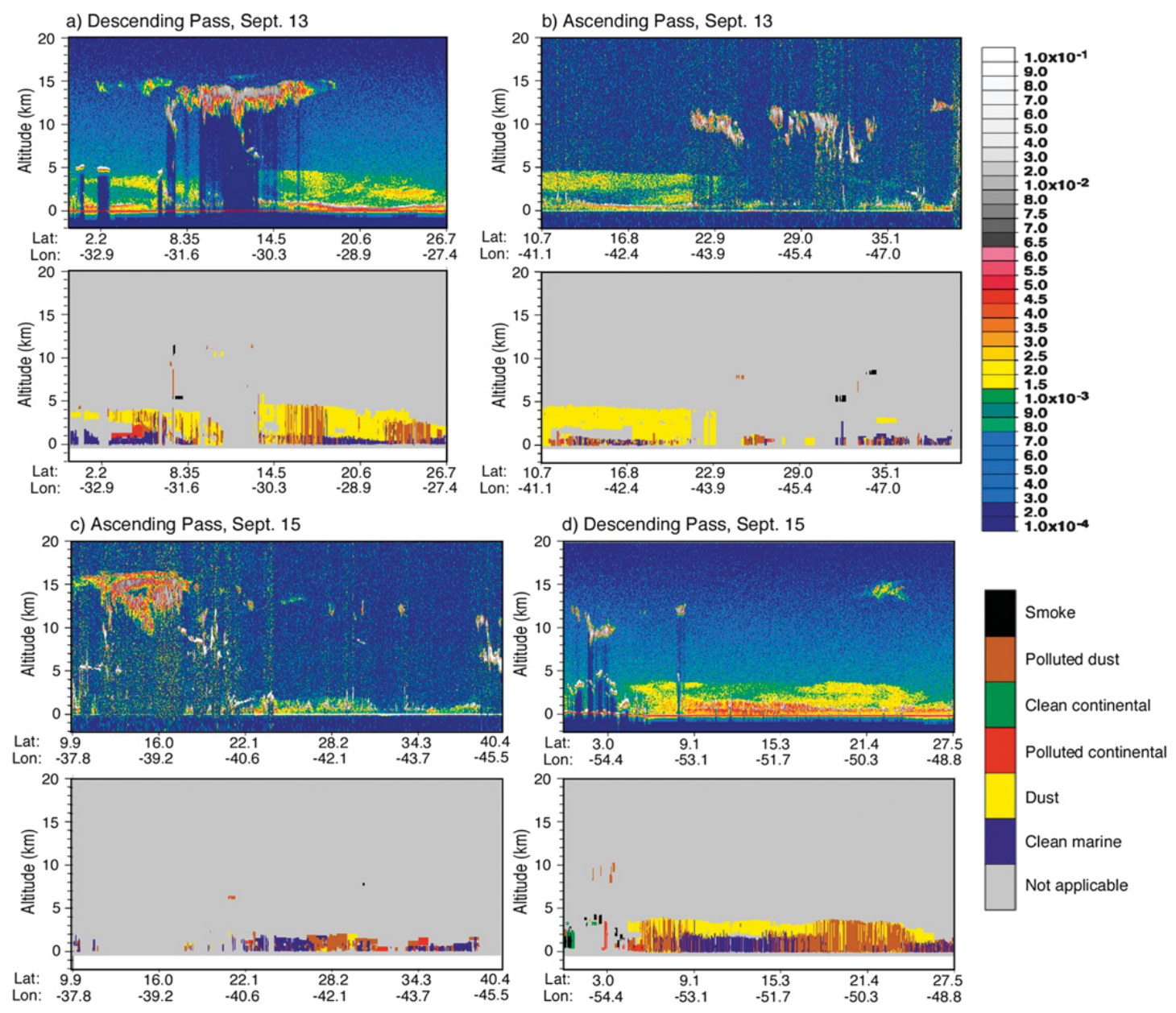

FIG. 9. CALIPSO (top) aerosol backscatter and (bottom) aerosol type for approximately (a) 0353 UTC 13 Sep, (b) 1626 UTC $13 \mathrm{Sep}$, (c) 1614 UTC 15 Sep, and (d) 0520 UTC 15 Sep. Locations of cross sections are shown in Fig. 2. In addition, the location of (a) is shown in Fig. 8 and locations of (c) and (d) in Fig. 11.

the AIRS and dropsonde measurements, which may explain the discrepancy. Despite the time difference, the $\mathrm{RH}$ profile is reasonably good in terms of showing that the driest air is above $600 \mathrm{hPa}$ and therefore is not associated with the SAL.

Dropsondes in the very dry air on the western side of the storm (S1-S6) all show a very deep dry layer from about 800 or $850 \mathrm{hPa}$ and above. Dropsondes S1-S4 indicate wind speed maxima near $800 \mathrm{hPa}(700 \mathrm{hPa}$ for S2), which is low for a typical AEJ whose wind speed maximum is usually closer to $700-600 \mathrm{hPa}$ (Burpee 1972; Reed et al. 1977). Profile S2 probably most resembles a SAL profile, with its $700-\mathrm{hPa}$ wind speed maximum, the increase in $\mathrm{RH}$ between 825 and $700 \mathrm{hPa}$, and temperature inversions at the base $(\sim 850 \mathrm{hPa})$ and top $(\sim 700 \mathrm{hPa})$ of the SAL. Profile S4 is qualitatively similar to S2, but with a wind maximum closer to $800 \mathrm{hPa}$, only a slight increase in RH between 725 and $650 \mathrm{hPa}$, and a distinct lack of temperature inversions at the base and top of the SAL. Other profiles such as S5 and S6, immediately adjacent to
Helene, exhibit no midlevel wind speed maximum and show a deep layer of very dry air, with a possible moist detrainment layer near $600 \mathrm{hPa}$ in S5. Following the dry tongue wrapping around the southern and eastern sides of Helene, dropsondes S10-S15 and the AIRS data indicate that the major dry layer was at middle to upper levels, with the humidity minimum above $500 \mathrm{hPa}$ rather than down at lower levels were SAL air to be wrapping around. These results suggest that the dry-air intrusion around the southern and eastern sides of the storm at this time may have been related more to subsidence of mid-to-upperlevel dry air rather than the SAL, although some SAL air is likely also present.

\section{b. Protection of the core and intensity change}

The figures above suggest that the SAL air mass seen around the northern and western sides of the storm at early stages (12-13 September) was gradually replaced by a much drier and largely dust-free (above the boundary layer) source of dry air around $14-15$ September. This 

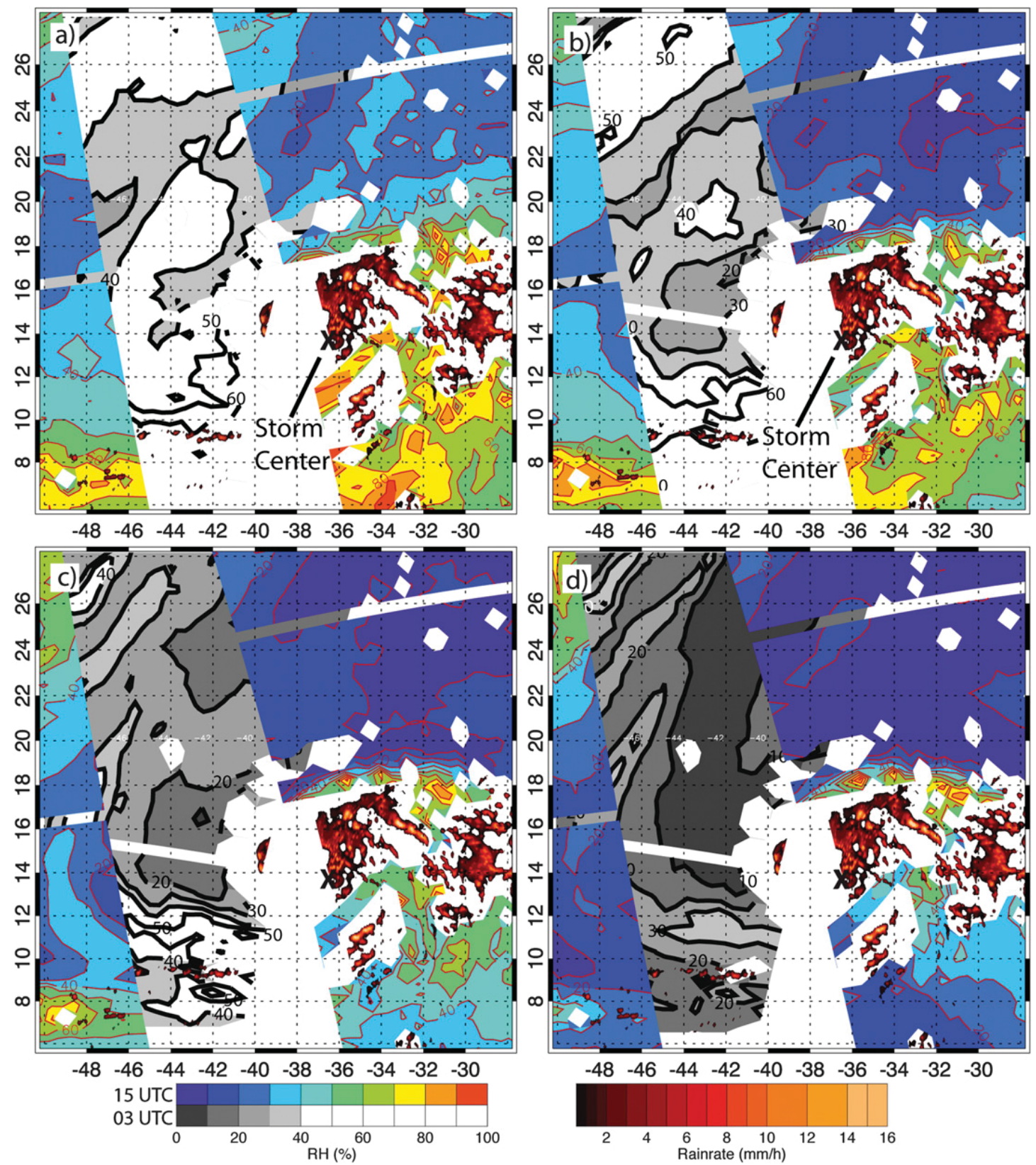

FIG. 10. As in Fig. 4, but for a merged analysis of data from $\sim 0300$ UTC (RH in gray shading) and $\sim 1500$ UTC (RH in color shading) 14 Sep. The analysis is valid for 1500 UTC, with 0300 UTC data shifted to account for storm motion. The left color scale shows RH contour values for both times. The estimated center of Helene from the NHC is indicated by the $\times$.

dry air wrapped around the storm to its eastern side by 15-16 September. An important question is, what role did this dry air play in the early intensity evolution of Helene?

Dunkerton et al. (2009) developed the concept of a protective wave "pouch" that protects an incipient vortex from deleterious influences of its environment. In a reference frame moving with the parent wave disturbance, a recirculation region within the critical layer of the parent wave is associated with cyclonic rotation and weak straining deformation. This region provides a set of approximately closed material contours within which air tends to be repeatedly moistened by convection and protected to some degree from intrusions of dry air and deformation by horizontal or vertical shear. As a tropical system forms and strengthens, the higher vorticity enhances the protection of the inner-core region and increases the vertical-wind shear threshold needed for environmental interaction (Riemer and Montgomery 2011).

Figure 13 shows a zoomed-in view of the MODIS AOD values for the period 12-15 September, overlaid 


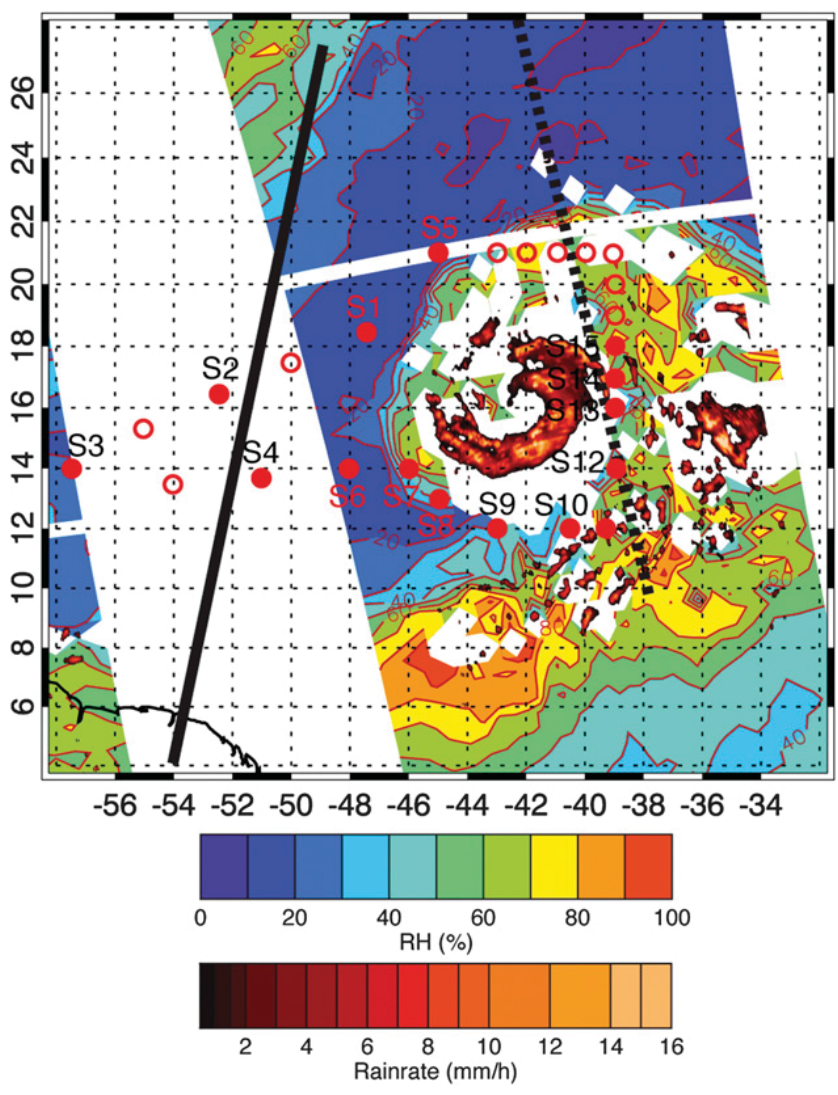

FIG. 11. As in Fig. 4, but for $\sim 1600$ UTC 15 Sep for the 700 600 -hPa layer. The black dotted line shows the position of the coincident CALIPSO overpass in Fig. 9c while the solid line shows the CALIPSO cross section $12 \mathrm{~h}$ earlier in Fig. 9d. Red circles indicate the locations of dropsondes from the NOAA G-IV with red filling indicating soundings shown in Fig. 12.

with contours of $\mathrm{RH}(\leq 50 \%)$ and storm-relative streamlines averaged over the 850-700-hPa layer from the 1200 UTC ERA-Interim analyses (similar results are obtained with NCEP and MERRA analyses). Also shown in the figure are the Geostationary Operational Environmental Satellite (GOES) visible images and high-frequency polarization corrected temperatures from either TRMM, Special Sensor Microwave Imager (SSM/I), or AMSR-E at times closest to 1200 UTC and adjusted for storm motion. As described by Dunkerton et al. (2009), the system-relative streamlines are better suited to detecting the likely pathways of entrainment of dry and dusty air than those in a ground-relative reference frame. Although gaps in the MODIS data preclude a detailed depiction of the dust structure, the general evolution of the dust layer during this period is clear.

On 12 September (Fig. 13a), a concentrated region of dust was collocated with a zone of strong northeasterly flow associated with the AEJ. The dust was just north of TD 8 and appeared to have a sharp southern boundary, as seen just northeastward of the convective system. The cloud system was enclosed within a weak pouch at midlevels in an environment with substantial $850-500-\mathrm{hPa}$ easterly shear, suggesting potential ingestion of SAL air in this layer on the western side of the storm. According to LASE and dropsonde measurements (Fig. 3), dust was plentiful at lower levels while conditions closer to the storm were generally moist, consistent with the ERA analysis showing $\mathrm{RH}>50 \%$ in this region. Jenkins and Pratt (2008) and Jenkins et al. (2008) suggested that the dust may have invigorated the convection although it is unclear how such convective invigoration impacted development of the tropical cyclone.

On 13 September (Fig. 13b), prior to the onset of any significant intensification (Fig. 1), a band of high AOD was found on the northwestern side of Helene close to the approximate dividing streamline. ERA-Interimderived RHs in this air were $\sim 30 \%-50 \%$ but quickly transitioned to more humid air near the cloud system, consistent with the AIRS retrievals in Fig. 8. In the nearly dust-free air well to the northeast, RHs were less than $30 \%$. Similar to the previous day, the winds in the dust layer were primarily moving tangential to Helene at this level, suggesting limited inflow of SAL air into the convective system at low to midlevels. Outside of the pouch, some dust was likely being transported around to the southern side of the storm, consistent with the CALIPSO data in Fig. 9a.

On 14 September, Helene began to slowly intensify (Fig. 1, from $\sim 15$ to $20 \mathrm{~m} \mathrm{~s}^{-1}$ ). Gaps in the MODIS data (Fig. 13c) make determination of the dust layer position and structure difficult. However, the data suggest that the dust was moving toward the southwestern side of Helene and was embedded in flow that lay mostly outside of the dividing streamline. The convection was largely confined within the region of the pouch. Relative humidities within the dust layer remained around $40 \%-$ $50 \%$. On the northwestern side of Helene, a tongue of air with $\mathrm{RH}<30 \%$ had begun to surge southward from the region of dry, nearly dust-free air to the north.

By 15 September, more significant storm intensification occurred as the maximum wind speed increased from 20 to $31 \mathrm{~m} \mathrm{~s}^{-1}$ (Fig. 1). During this day (Fig. 13d), the majority of the dust was clearly west of Helene, separated from the storm by a region of very dry, nearly dust-free (above the boundary layer) air moving southward along the western side of the Helene pouch. This dry air was beginning to wrap around the southern side of Helene, along with some remaining dusty air well to the southwest of the storm, but was still situated more than $400 \mathrm{~km}$ from the storm center where it would have very limited interaction with the inner core of the storm.

The large separation of the dry air from the center of Helene between 12 and 15 September may have limited 

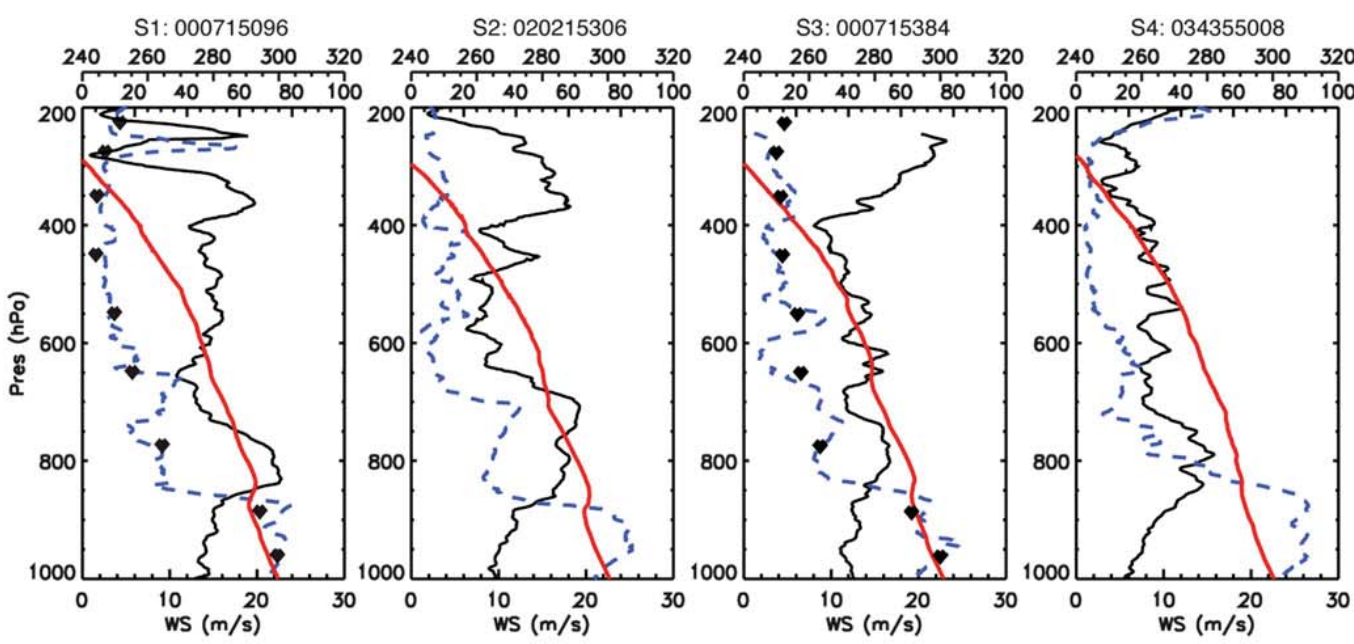

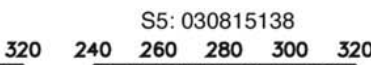

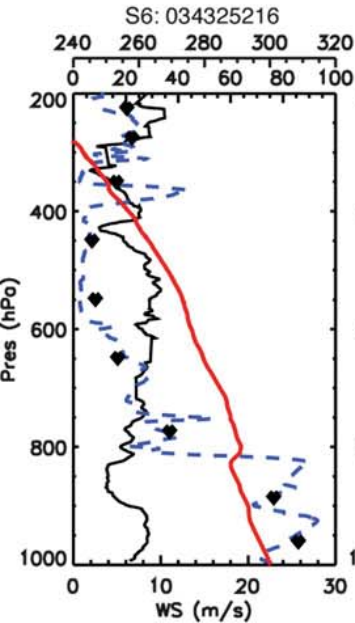

S7: 030815230

S8: 030725214

S9: 304135052
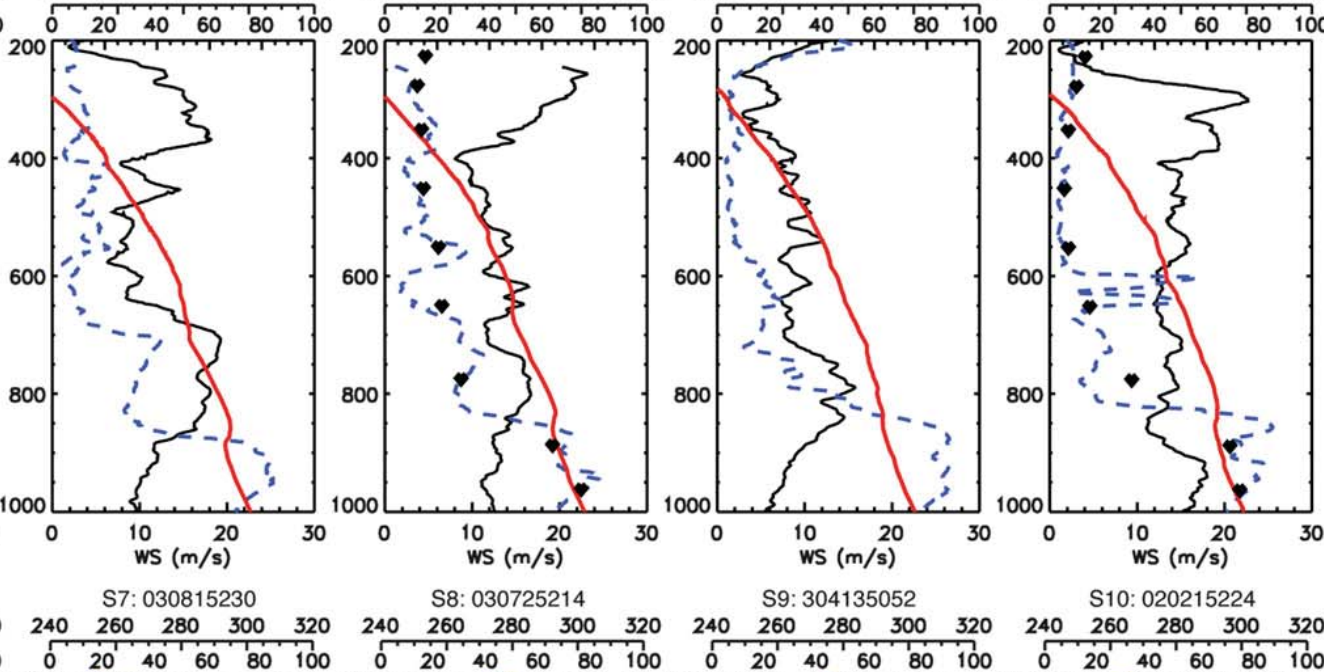

S10: 020215224
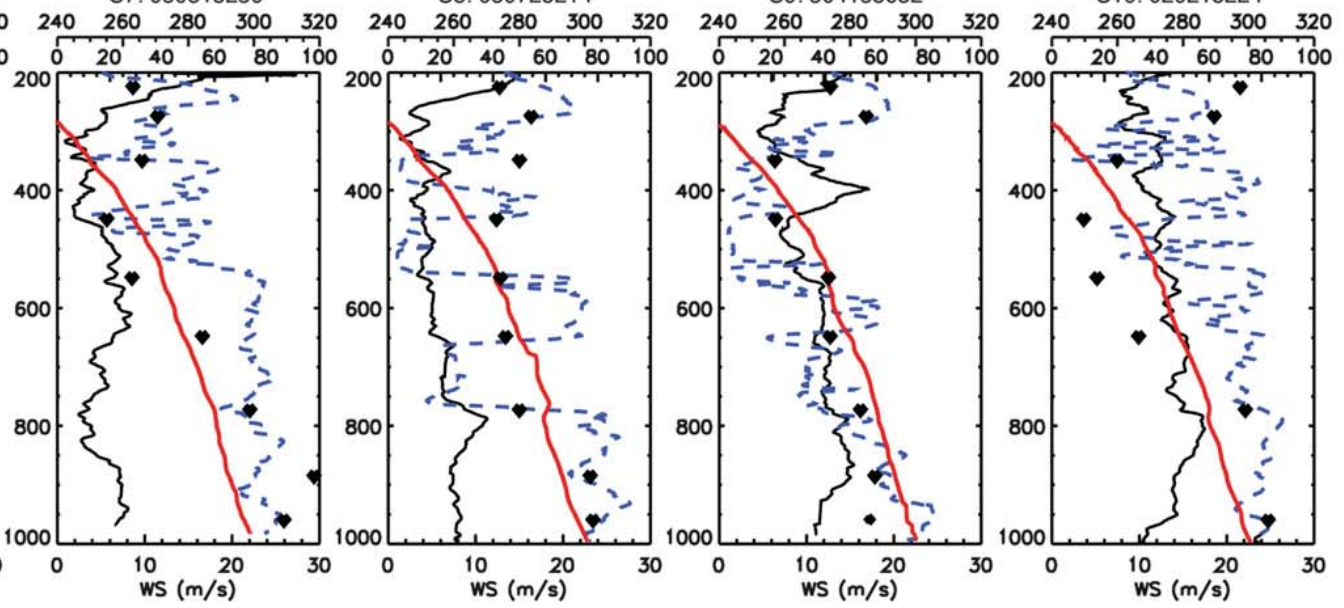

S11: 000715092

S12: 034325137

S13: 030725043
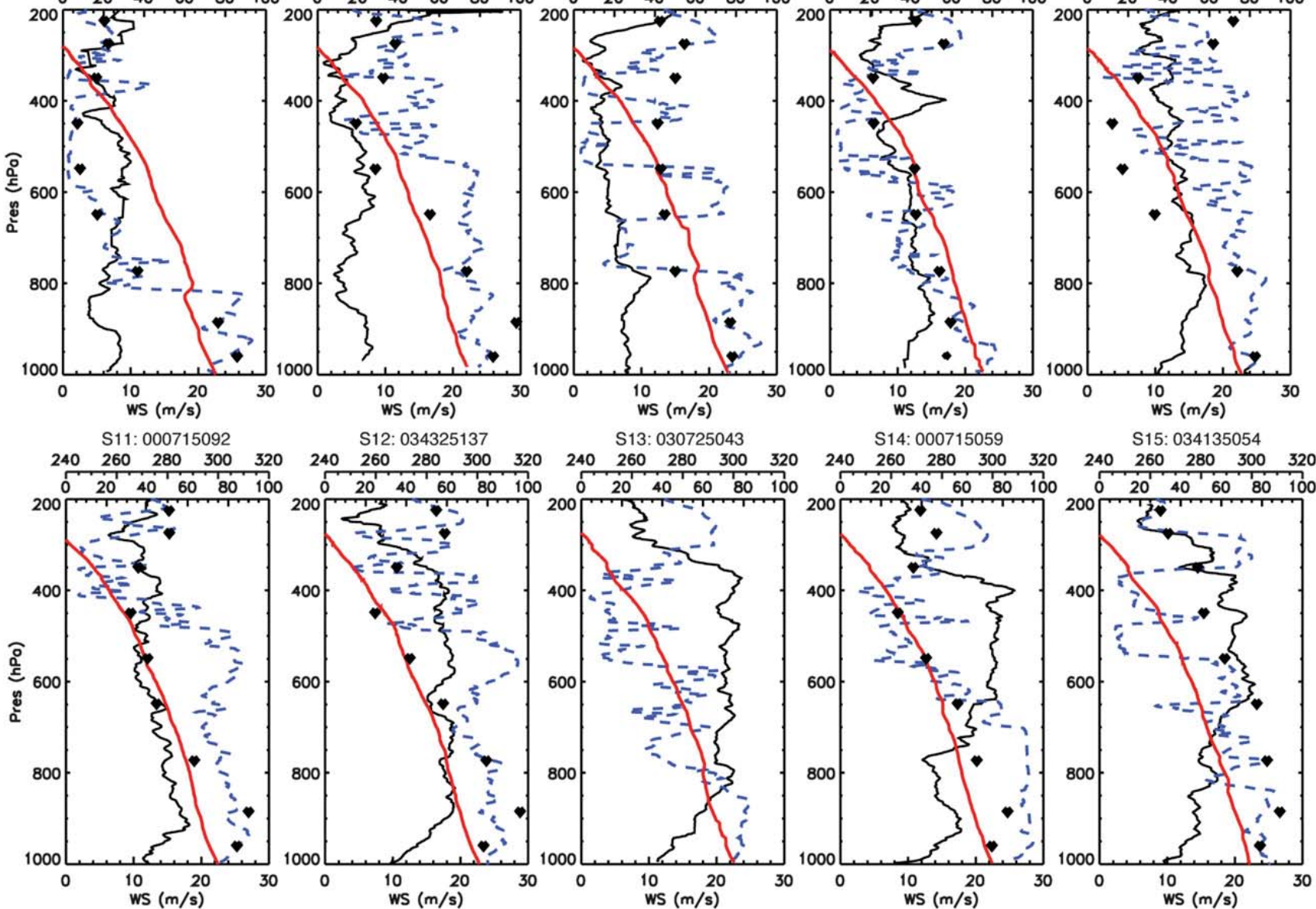

FIG. 12. Profiles of temperature (red lines), RH (blue dashed lines), and wind speed (black lines) for dropsondes indicated by filled circles in Fig. 11. Soundings are labeled from S1 to S15. The nine-digit numbers after the labels are the sounding identification numbers. Black symbols indicate the AIRS derived layer-mean RH, when available, plotted at the center of the layer.

the dry air's negative influence on Helene's intensification during this period. In an idealized modeling study of the impacts of dry midlevel air on storm intensification in environments of no mean flow, Braun et al. (2012) found that dry air needed to be within about
$270 \mathrm{~km}$ of the storm center in order to have any negative impact on storm intensification. During 12-13 September, when low-level shear was strongest, dry SAL air may have interacted with convection on the western edge of the developing storm, and so some influence 
(a)

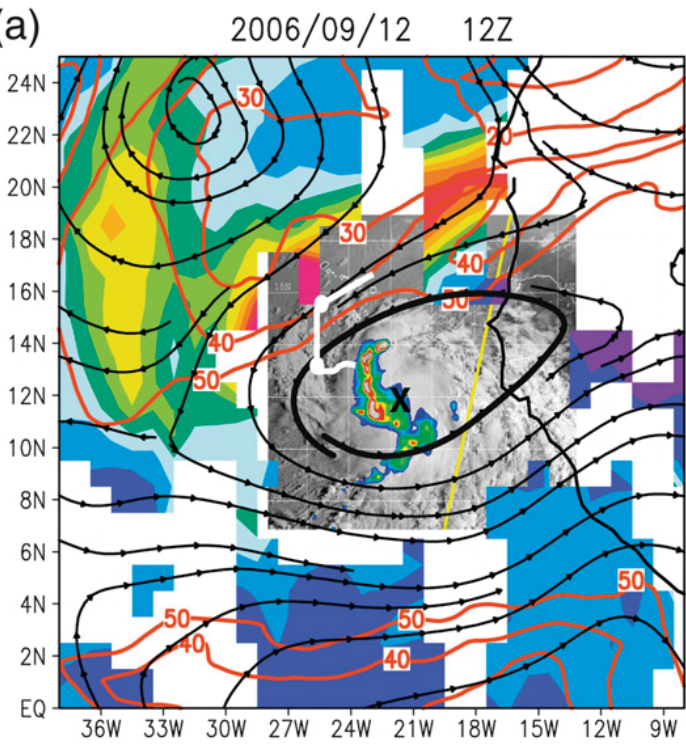

(b)

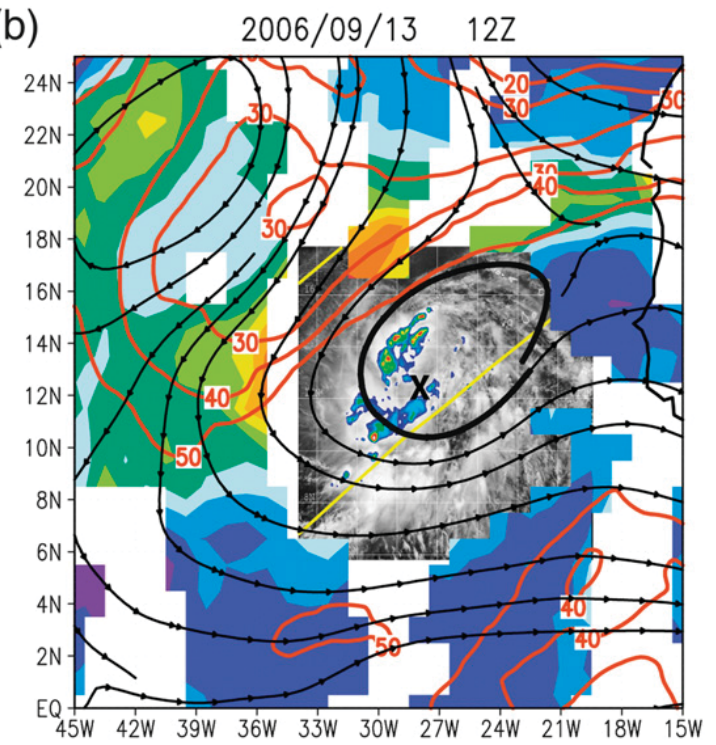

(c)

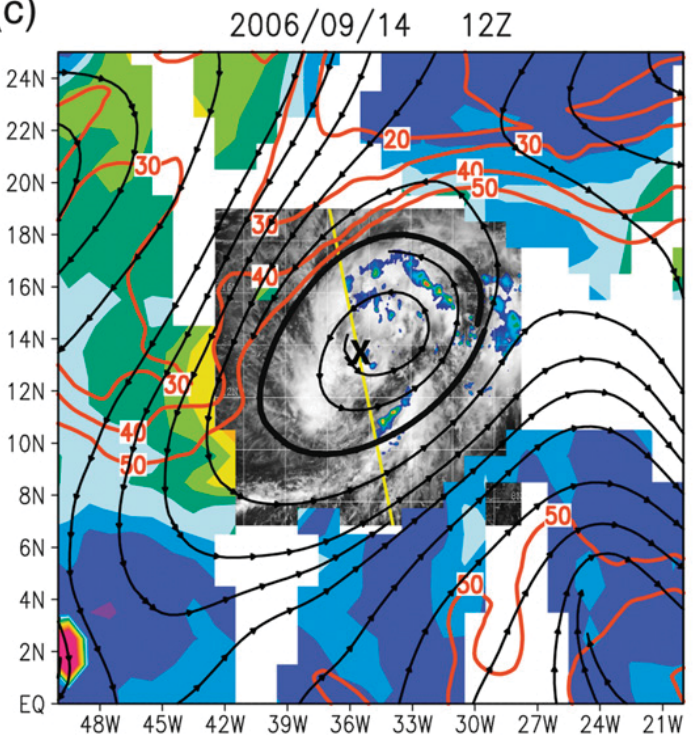

(d)

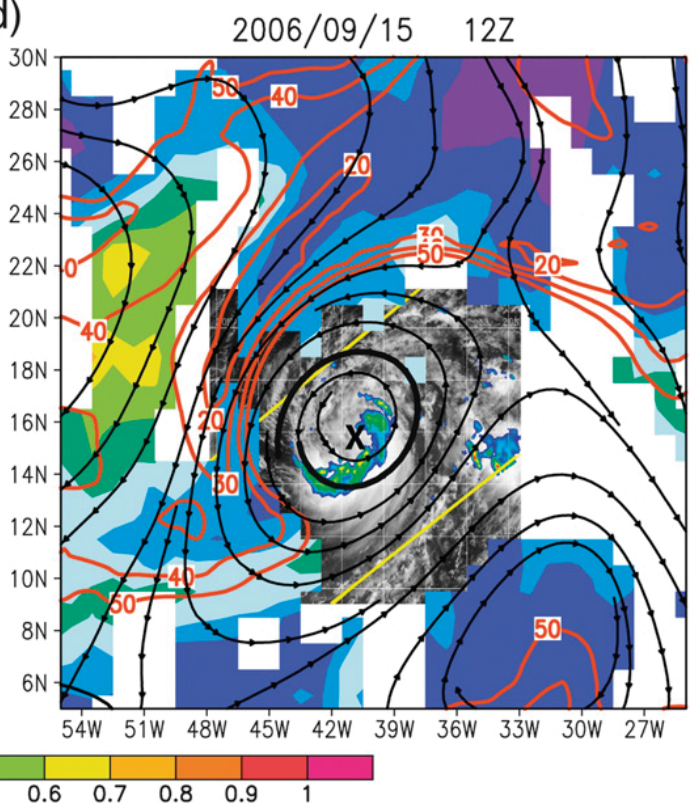

FIG. 13. MODIS AOD values valid for the indicated dates. White shading indicates missing data. Orange contours indicate $850-700-\mathrm{hPa}$ layer-averaged relative humidity less than $50 \%$ at $10 \%$ increments from the ERA-Interim analyses. Streamlines indicate storm-relative flow averaged over $850-700 \mathrm{hPa}$ from the ERA-Interim analyses, with storm motion estimated from best track data. The thick streamline indicates an approximate dividing streamline. Background images show GOES visible imagery and (a) SSM/I 91-GHz PCT at 1001 UTC, (b) TRMM 85-GHz PCT at 1445 UTC, (c) AMSR-E 89-GHz PCT at 1524 UTC, and (d) TRMM 85-GHz PCT at 1435 UTC for the respective dates. Thin yellow lines mark the edges of the microwave swaths, and in (b) and (c) only partial coverage of the innercore precipitation is available. GOES and microwave imagery were provided by the Naval Research Laboratory tropical cyclone website (http://www.nrlmry.navy.mil/tc_pages/tc_home.html). The short white line segment and two circles in (a) show the locations of the LASE data and two dropsondes in Fig. 3. The estimated center of Helene from the NHC is indicated by the $\times$.

cannot be ruled out given the very small intensification that occurred during this period. During 14-17 September, the dry air wrapped extensively around Helene as it intensified into a major hurricane. Since vertical wind shear was weak, the findings of Braun et al. (2012) are likely valid and suggest that the dry air acted only as a minor impediment to intensification. Also, as shown in Fig. 8 of Riemer and Montgomery (2011), the very weak mean storm-relative flow $\left(\sim 1-2 \mathrm{~m} \mathrm{~s}^{-1}\right.$ or half the value of the deep-layer vertical shear in Fig. 1) implies a small 
interaction of the rainbands with their environment, with that interaction decreasing over time as the storm intensified. Finally, these results are consistent with the finding by Tang and Emanuel (2012) that little ventilation of storms occurs in the absence of significant vertical shear.

\section{c. Global analyses and trajectories}

This section uses trajectories calculated from the NCEP and ERA-Interim analyses to show that the SAL air originally surrounding the northern and western sides of the storm at early stages (12-13 September) was gradually replaced by a non-SAL source of dry air that became the dominant dry-air feature. The trajectories show that this dry air was a mixture of air of both subtropical African origin and midlatitude origin, but that its defining characteristic was the substantial large-scale subsidence that produced it. While differences do exist between the analyses, their representation of the SAL is comparable in terms of SAL depth and structure. Similar to the DC8 soundings in Fig. 3, the NCEP, ERAInterim, and MERRA analyses also depict a vertical structure of the SAL with RH increasing with height as the SAL air mass came off of Africa and moved northward and eventually westward of Helene. Figure 14 shows north-south vertical cross sections through the wave axis at 1200 UTC 12 September from the three analyses. In each, the RH is characterized by extremely dry air $(<30 \%)$ within the lower part of the SAL near $900 \mathrm{hPa}$ and then increasing $\mathrm{RH}$ with height with a midlevel maximum of $\sim 50 \%-65 \%$ at the top of the SAL near $600 \mathrm{hPa}$. This $600-\mathrm{hPa}$ top is consistent with the LASE and CALIPSO-observed dust tops of $\sim 4.7 \mathrm{~km}(\sim 575 \mathrm{hPa})$ or lower (Figs. 3 and 9). Relative humidity values above the SAL are very dry with values typically below $30 \%$. The comparable structures of the AEJ suggest that temperature gradients associated with the SAL are also similar among the analyses.

Six-day backward trajectories were calculated for several levels and times in order to determine the origins of the air around Helene and the role of the SAL versus subsidence in producing the dry air wrapping around Helene. Results for $700 \mathrm{hPa}$ for $14-16$ September using both ERA-Interim and NCEP analyses are presented here. Figure 15 shows objectively identified SAL regions based upon the criteria specified in section $2 \mathrm{c}$ while Fig. 16 depicts the total descent along trajectories during the 6-day period. Both figures also show overlaid contours of $\mathrm{RH}$ for values $\leq 50 \%$.

Trajectories from ERA-Interim and NCEP global analyses (Fig. 15) demonstrate the SAL origin of the dry air mass to the west and immediately north of Helene on 14 September and very dry non-SAL air farther north of Helene. The boundaries of the identified SAL air are in
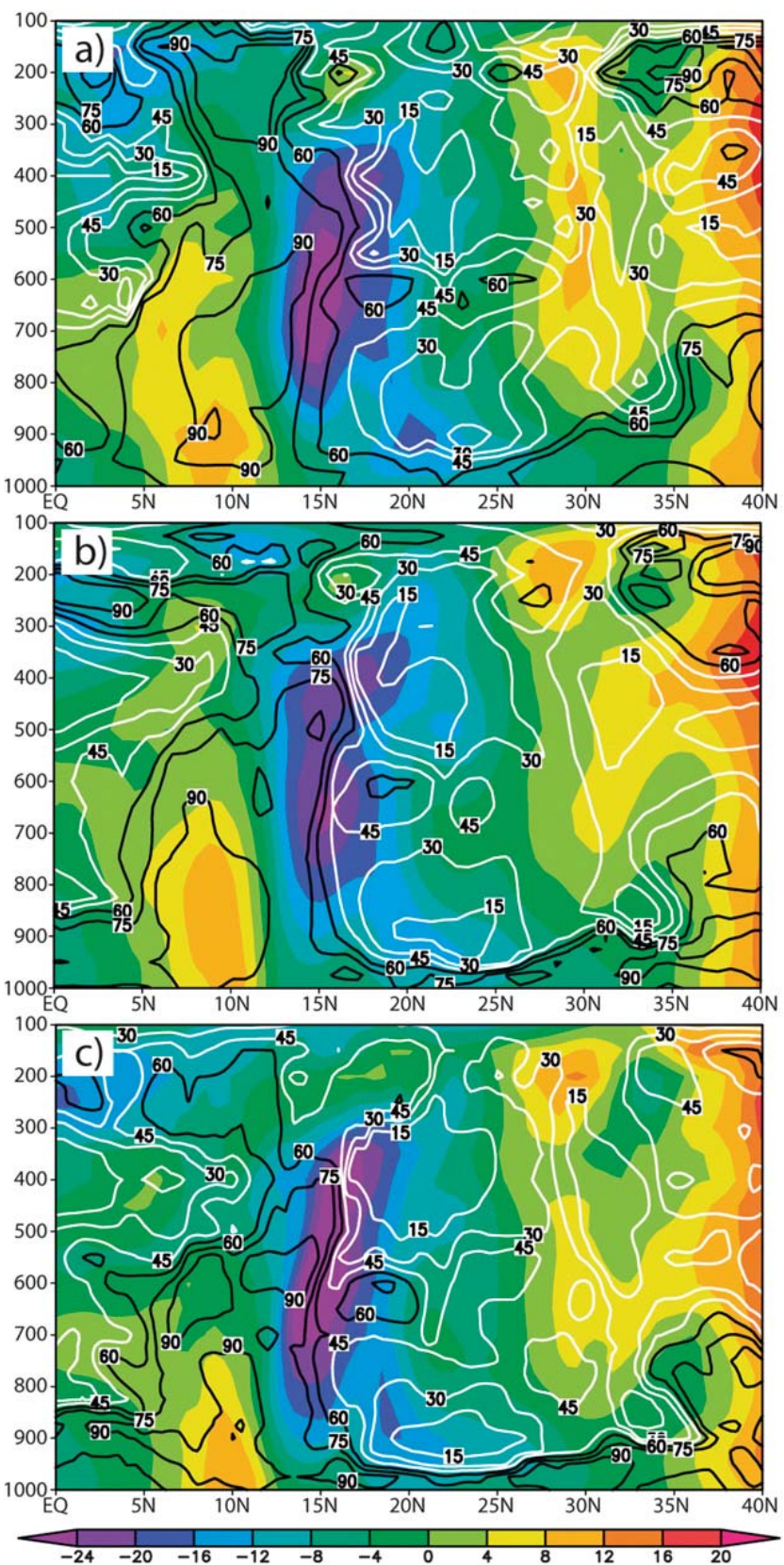

FIG. 14. South-north vertical cross sections of zonal velocity and $\mathrm{RH}$ along the pre-Helene wave axis $\left(23^{\circ} \mathrm{W}\right.$ longitude $)$ at $1200 \mathrm{UTC}$ 12 Sep from (a) NCEP, (b) ERA-Interim, and (c) MERRA. Zonal velocity is shaded $\left(\mathrm{m} \mathrm{s}^{-1}\right)$ with negative values indicating easterly winds. RH is plotted at $15 \%$ increments starting at $15 \%$, with black contours for $\mathrm{RH} \geq 60 \%$ and white contours for $\mathrm{RH} \leq 45 \%$.

reasonably good agreement with MODIS dust boundaries (bold lines). ${ }^{5}$ Both analyses also capture the gradual erosion or replacement of the SAL air over the

\footnotetext{
${ }^{5}$ Note that the MODIS boundary delineates dust that may be present at any height in the column whereas the trajectories are for a specific level, so one-to-one correspondence at a given level is not expected.
} 



FIG. 15. Diagnosed regions of SAL air at $700 \mathrm{hPa}$ (see section 2c) from 6-day backward air trajectories, with orange shading indicating locations of likely SAL air, light blue indicating air from the Saharan region but from above the SAL: 1200 UTC (a),(b) 14 Sep, (c),(d) 15 Sep, and (e),(f) 16 Sep. Black contours in all panels represent 700-hPa relative humidity at $10 \%$ intervals up to $50 \%$ to show locations of dry air for each day. Calculations are from the (left) ERA-Interim and (right) NCEP global analyses. The black circles indicate the center locations of Hurricane Helene. Bold red lines indicate approximate MODIS dust boundaries, dashed where approximated because of areas of missing data.

next couple of days by air from a non-SAL source and the wrapping of a dry-air tongue around to the eastern side of Helene, consistent with the AIRS data. However, the NCEP RH values in this dry tongue are $\sim 10 \%-20 \%$ higher than in the ERA analysis on 16 September, so RH values $<50 \%$ only reach the southern side of the storm.

On 16 September, the ERA trajectory analysis (Fig. 15e) suggests a mesoscale region of SAL air with $\mathrm{RH}>50 \%$ just north of the center while the NCEP analysis (Fig. 15f) shows only a very small region of SAL air. In the ERA case, analysis of the back trajectories indicates that this air corresponded to a region of SAL air on 12 September (Fig. 4a) that originated between 900 and $750 \mathrm{hPa}$ and was located just north of the storm at $16^{\circ}-20^{\circ} \mathrm{N}, 16^{\circ}-20^{\circ} \mathrm{W}$. This air on 12 September was characterized by $\mathrm{RH}$ values between $40 \%$ and $50 \%$ in the $850-700-\mathrm{hPa}$ layer (Fig. 4a) in 

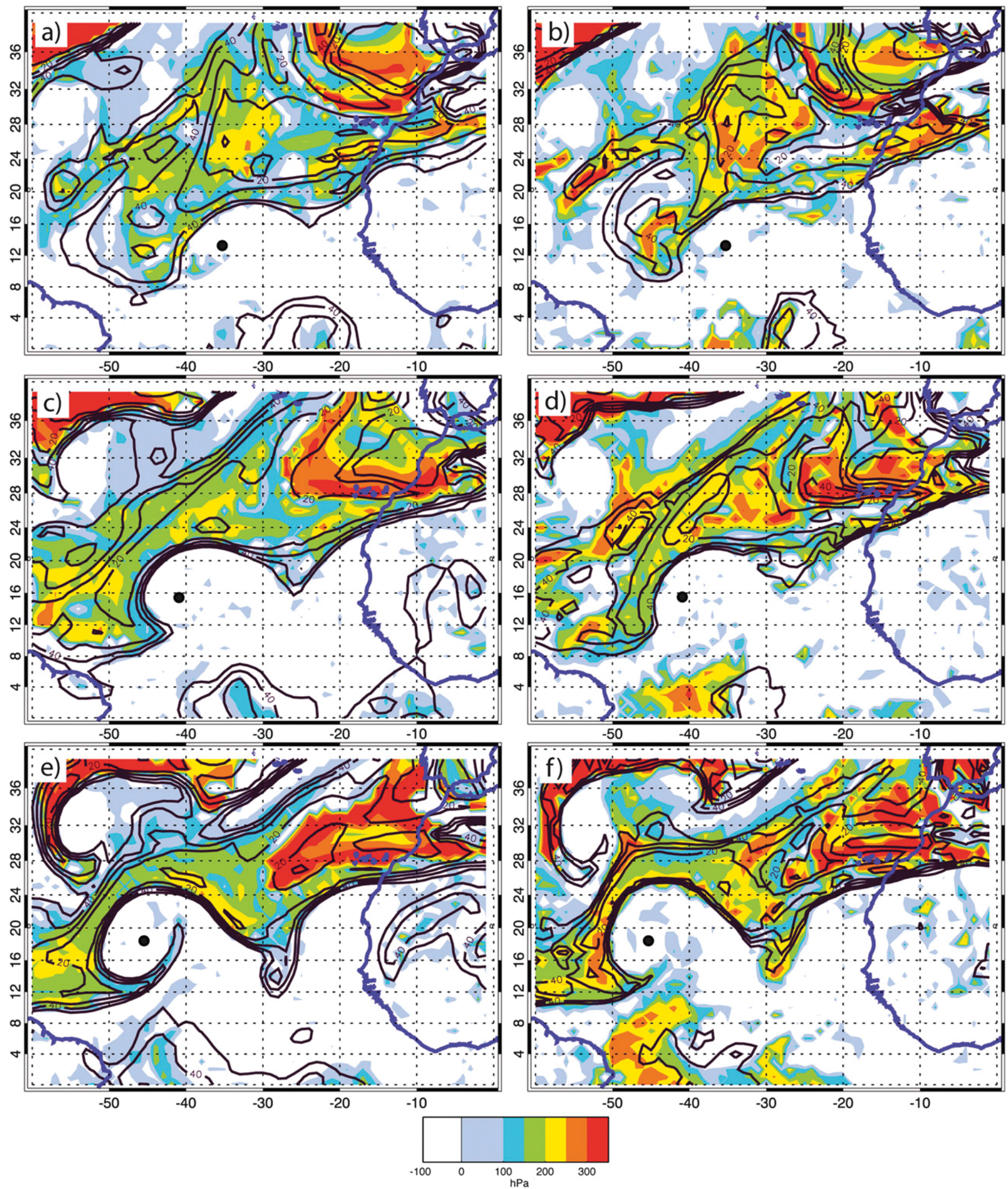

FIG. 16. Trajectory-based diagnosis of net subsidence (pressure change) along the trajectory paths, with positive values indicating total descent over 6 days ending at $700 \mathrm{hPa} 1200 \mathrm{UTC}$ (a),(b) $14 \mathrm{Sep}$, (c),(d) $15 \mathrm{Sep}$, and (e),(f) $16 \mathrm{Sep}$. Black contours in all panels represent $700-\mathrm{hPa}$ relative humidity at $10 \%$ intervals up to $50 \%$ to show locations of dry air for each day. Calculations are from the (left) ERAInterim and (right) NCEP global analyses. The black circles indicate the center locations of Hurricane Helene. 
the AIRS data and between $40 \%$ and $60 \%$ in the ERA analyses (not shown). By 14 September, this SAL air was on the western side of the storm (Fig. 15a; between the center of the storm and the $50 \% \mathrm{RH}$ contour to the west), but had moistened as a result of ascent so that humidities were between $50 \%$ and $90 \%$ in the ERA analyses. Figure 13c indicates that this region to the west of the center was occupied by a band of deep convective clouds, so it is likely that this SAL air was actually limited to the region just radially outside of this cloud band, consistent with earlier observations (Figs. 4a, 6d, and 8a). As a result, the extent of the SAL air within the moist portion of the vortex in Fig. 15a appears to be exaggerated and the same may be true for later times (Figs. 15c,e) as well. However, these results suggest that some Saharan air may have mixed into the inner region of Helene during 14-16 September, but may have been moistened during transit into the storm region.

The patterns of trajectory descent (Fig. 16) clearly indicate that the dry air masses were dominated by subsidence with parcels descending over the previous six days by as much as $100-300 \mathrm{hPa}$. By 15 September (Figs. 16c,d), the subsidence became pronounced along the western flank of the storm and by 16 September (Figs. 16e,f), the subsiding air wrapped around the southern and eastern sides of Helene. The subsidence wrapping south and east of Helene is more pronounced in the ERA than in the NCEP analysis, consistent with the lower relative humidity in the ERA analysis. The trajectory based descent estimates are consistent with the large-scale drying of the midlevels observed in the AIRS data and confirm the role of subsidence as a key source of dry air around Helene beginning 14 September.

\section{Intensification to major hurricane}

From 15 to 18 September, Hurricane Helene underwent steady intensification (Fig. 1), including a period of rapid intensification $\left(\sim 15 \mathrm{~m} \mathrm{~s}^{-1}\right.$ in $\left.24 \mathrm{~h}\right)$ on 17 September. Figure 17 shows the evolution of the RH in the 700 600-hPa layer from AIRS and the rainfall rate from AMSR-E during 16-18 September. By $\sim 0400$ UTC 16 September (Fig. 17a), very dry air $(<30 \%)$ had moved around to the eastern side of the storm and $\mathrm{RH}<60 \%$ was found as far around as the northern side of the rainbands. The precipitation was asymmetric, with most of the precipitation on the northeastern side of the storm. The asymmetry was likely associated with weak-to-moderate westerly wind shear $\left(\sim 4 \mathrm{~m} \mathrm{~s}^{-1}\right.$, Fig. 1a), but may also have been related to the intrusion of dry air (Braun et al. 2012). An eyewall was not yet apparent in the rainfall pattern. By 1700 UTC 16 September (Fig. 17b), a well-defined eyewall was clearly present in the AMSR-E data, with heavy precipitation on the northwestern side of the storm and an outer rainband along the southern half of the storm. Unfortunately, the AIRS data did not cover the dry tongue of air on the eastern side of the storm. However, $\mathrm{RH}$ values of $\sim 50 \%$ were seen on the western side close to the outer rainband.

A CALIPSO overpass at this time along the western side of the storm (Fig. 18, location shown in Fig. 17b) detected only a shallow layer of aerosols, generally below $2-\mathrm{km}$ altitude $(\sim 800 \mathrm{hPa})$, and containing a mixture of dust, marine aerosols, and polluted continental air. Dropsondes from the NOAA G-IV on this day (not shown) indicated a deep layer of dry air above $\sim 1.5 \mathrm{~km}$ and moist conditions below. Thus, the dry air observed by AIRS in the 700-600-hPa layer (Fig. 17b) was well above the aerosol layer while the less dry (by $\sim 10 \%$ ) 850-700-hPa layer (not shown) was just above the dust layer or included only the uppermost portion of the dust layer. Given the diagnosed patterns of subsidence in this region (section 3b; Fig. 16), the CALIPSO and AIRS data suggest that this dry layer was related to subsidence rather than the SAL.

By 0500 UTC 17 September (Fig. 17c), the dry air continued to extend into the eastern portion of the storm. A direct overpass by CALIPSO (Fig. 18b) shows a shallow layer of aerosols on the southern side of the storm, with mostly polluted continental and maritime aerosols south of $10^{\circ} \mathrm{N}$, but with a narrow zone of dust coincident with the lower portion of the dry tongue nearly $600 \mathrm{~km}$ to the south of the storm. Clearly, SAL air was present in the lower layers $(<2 \mathrm{~km})$ of this feature while non-SAL dry air lay above. Despite the presence of the dry air, Helene was undergoing rapid intensification and had a very clear and more symmetric eyewall structure.

By 1700 UTC 18 September (Fig. 17d), the RH field around Helene was becoming axisymmetrized since the dry tongue was much less visible and the storm was surrounded by a field of moderate $(50 \%-80 \%) \mathrm{RH}$. A well-defined eyewall was present and the precipitation region had expanded as numerous outer rainbands had formed. Around this time, intensification stopped and the storm subsequently weakened slowly. The next section examines the possible causes of this weakening of the storm.

\section{Weakening phase}

There were several potential factors that may have contributed to the end of intensification and the slow weakening of Helene beginning on 18 September, including secondary eyewall formation and eyewall replacement, increased vertical wind shear, and the movement over colder underlying ocean temperatures due to storm-induced 

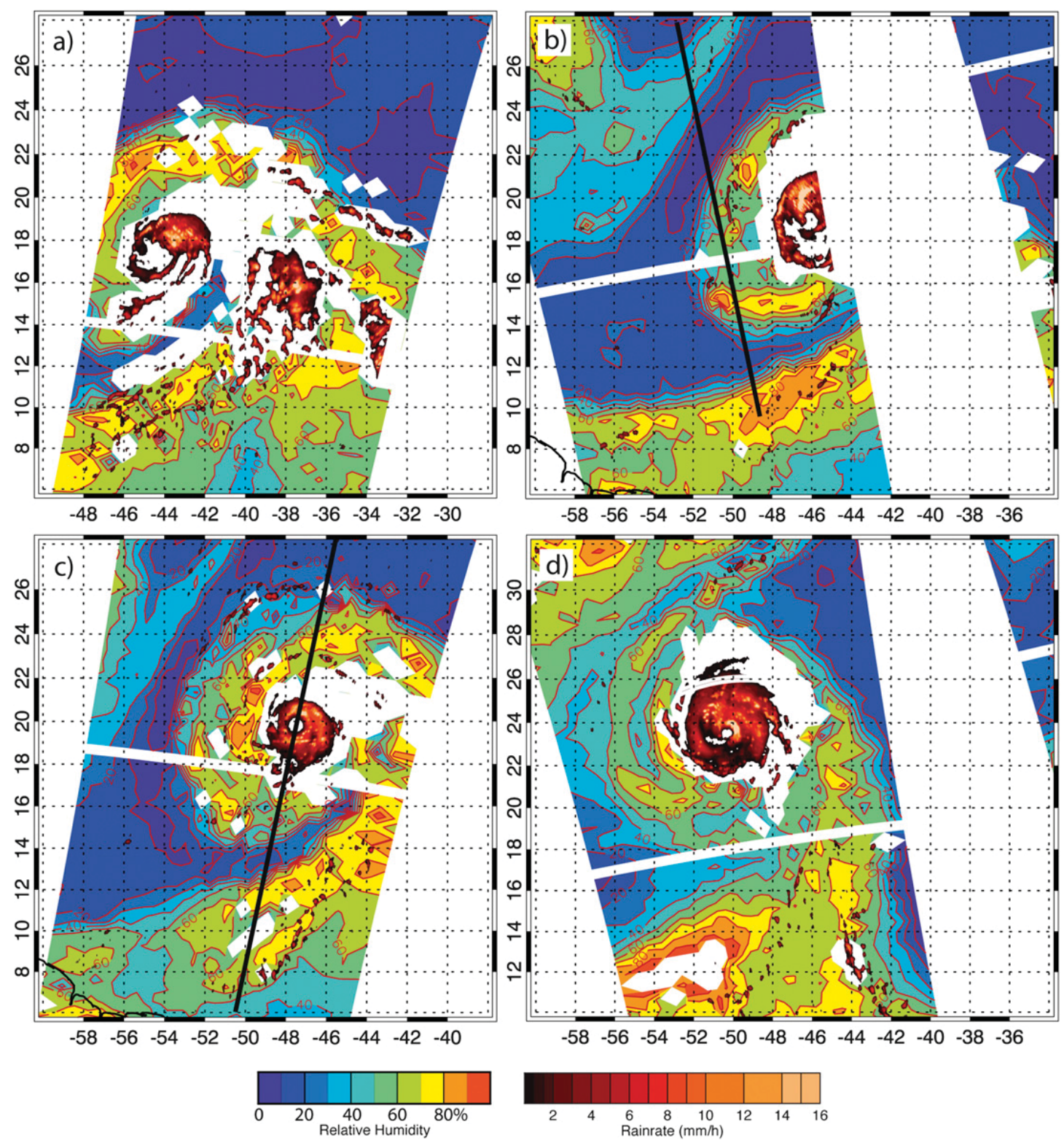

FIG. 17. AIRS/AMSU 700-600-hPa layer-averaged RH (left color scale) for (a) 0420 UTC 16 Sep, (b) 1650 UTC $16 \mathrm{Sep}$, (c) 0502 UTC $17 \mathrm{Sep}$, and (d) 1641 UTC 18 Sep. Superimposed within AIRS data gaps are the coincident AMSR-E derived surface rainfall estimates (right color scale). The black solid lines in (b) and (c) show the positions of the coincident CALIPSO overpasses in Figs. 18a and 18b, respectively.

cooling. In terms of vertical wind shear, Fig. 1a shows that large-scale deep-layer shear remained $<6 \mathrm{~m} \mathrm{~s}^{-1}$ through the intensification period and up to 4 days after Helene began to weaken. Only after 1200 UTC 22 September did shear increase markedly as the storm began extratropical transition. Hence, vertical shear was likely not a key cause of the weakening that began on 18 September.

As mentioned by Franklin and Brown (2008), concentric eyewall formation contributed to the weakening of Helene. Figure 19 shows a series of rainfall estimates from passive microwave imagery beginning late on 17
September and ending on 20 September. On 17 September (Fig. 19a), during rapid intensification, the rainfall pattern was highly asymmetric likely as a result of modest (4-6 $\mathrm{m} \mathrm{s}^{-1}$ ) vertical shear at this time. By midday on 18 September (Fig. 19b), outer rainband precipitation increased on the northern and western sides of Helene and became even more pronounced later in the day (Fig. 19c). The eyewall remained highly asymmetric, but with the rainfall having shifted from the eastern to the northern side of the eyewall. By 19 September (Fig. 19d), the outer band surrounded the asymmetric eyewall and by later that day (Fig. 19e), there was evidence of contraction of 
a) Ascending Pass, Sept. 16

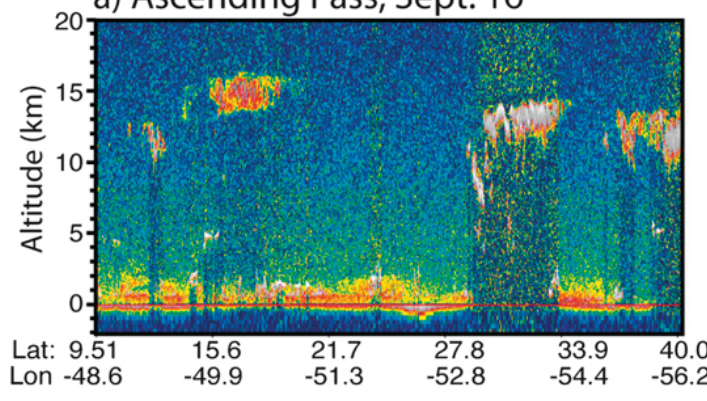

b) Descending Pass, Sept. 17

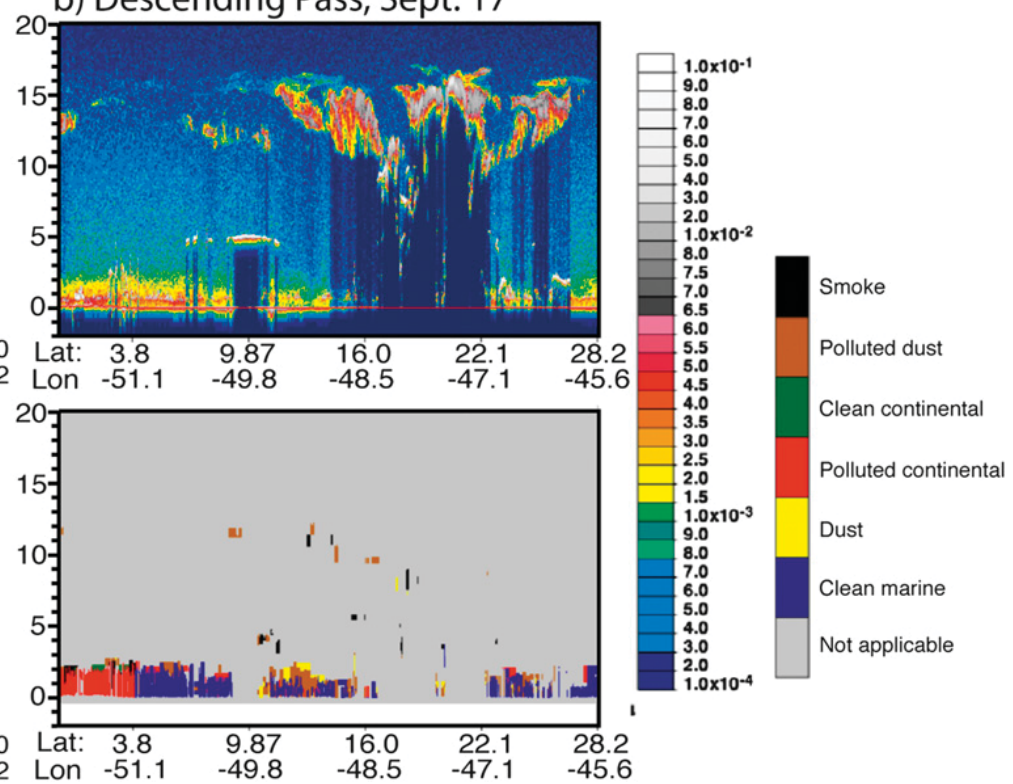

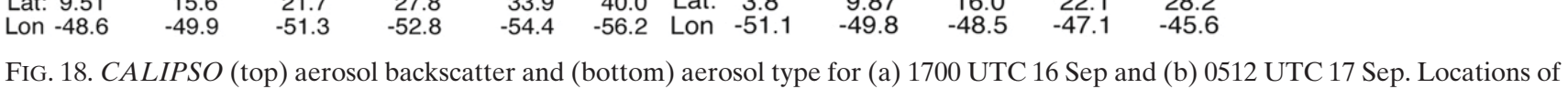
cross sections are shown in Figs. $17 \mathrm{~b}$ and $17 \mathrm{c}$.

the outer band. By 20 September (Fig. 19f), the original eyewall was diminishing and the outer convective rainband had become the dominant precipitation feature.

Concurrent with the eyewall replacement cycle, significant changes also occurred in the SST fields (Fig. 20). Helene moved along the northern edge of a pool of warm SSTs with temperatures $\sim 28^{\circ} \mathrm{C}$. As Helene intensified on 17 September (Fig. 20a), some cooling became evident on the northern side and in the wake of Helene, with SSTs near $26^{\circ} \mathrm{C}$. Beginning on 18 September, the cold wake began to expand and, by 19 September, a pronounced cold wake was present with SSTs $<26^{\circ} \mathrm{C}$ over a broad area (and as low as $23^{\circ} \mathrm{C}$ ). In addition, a prominent region of cooler temperatures was present to the north and northwest of Helene associated with the cold wake of Hurricane Gordon. Over the next several days (Figs. 20d-f), Helene moved over the wake of Gordon and also continued to produce its own wake. The development of the cold wake was likely tied to both Helene's intensification and a slowing of its forward motion. Prior to 15 September, Helene's storm motion exceeded $7 \mathrm{~m} \mathrm{~s}^{-1}$. Between 16 and 19 September, the storm motion decreased from 5.6 to $3.2 \mathrm{~m} \mathrm{~s}^{-1}$, suggesting the potential for increased impact of storm-induced cooling of the underlying ocean. Observationally, Brand (1971), Price (1981), Lin et al. (2009), Dare and McBride (2011), and Knaff et al. (2013) have shown that slower storm motions tend to produce greater reductions in SST. Simulations by Khain and Ginis (1991), Bender et al. (1993), and Bender and Ginis (2000) have yielded similar results and have shown that such cooling can have a significant impact in reducing storm intensity.

\section{Conclusions}

Previous studies on the impact of the Saharan air layer on tropical cyclone genesis and intensification have yielded mixed results, with some studies (Karyampudi and Carlson 1988; Karyampudi and Pierce 2002) suggesting that the SAL can have a positive influence on development and other studies (DV04; Jones et al. 2007; Shu and $\mathrm{Wu} 2009$ ) suggesting that the SAL may be a negative influence. The NAMMA field campaign conducted over the eastern Atlantic Ocean in 2006 generated considerable interest in SAL-tropical cyclone interactions (Lau and Kim 2007a; Jenkins and Pratt 2008; Jenkins et al. 2008; Vizy and Cook 2009; Ross et al. 2009; Zipser et al. 2009), with Hurricane Helene being viewed as a key example of this interaction. The present study examines the role of the SAL in the evolution of Helene using a suite of remote sensing data, aircraft observations, and global meteorological analyses, with a particular focus on the transition of the storm environment from one dominated by the SAL to one dominated by subsidenceinduced drying.

During the pre- and early intensification stages of Helene (12-15 September), significant Saharan dust (and SAL air) was present on the northern and western sides of the storm. The SAL air had a characteristic RH profile with RH increasing with height up to the SAL top 

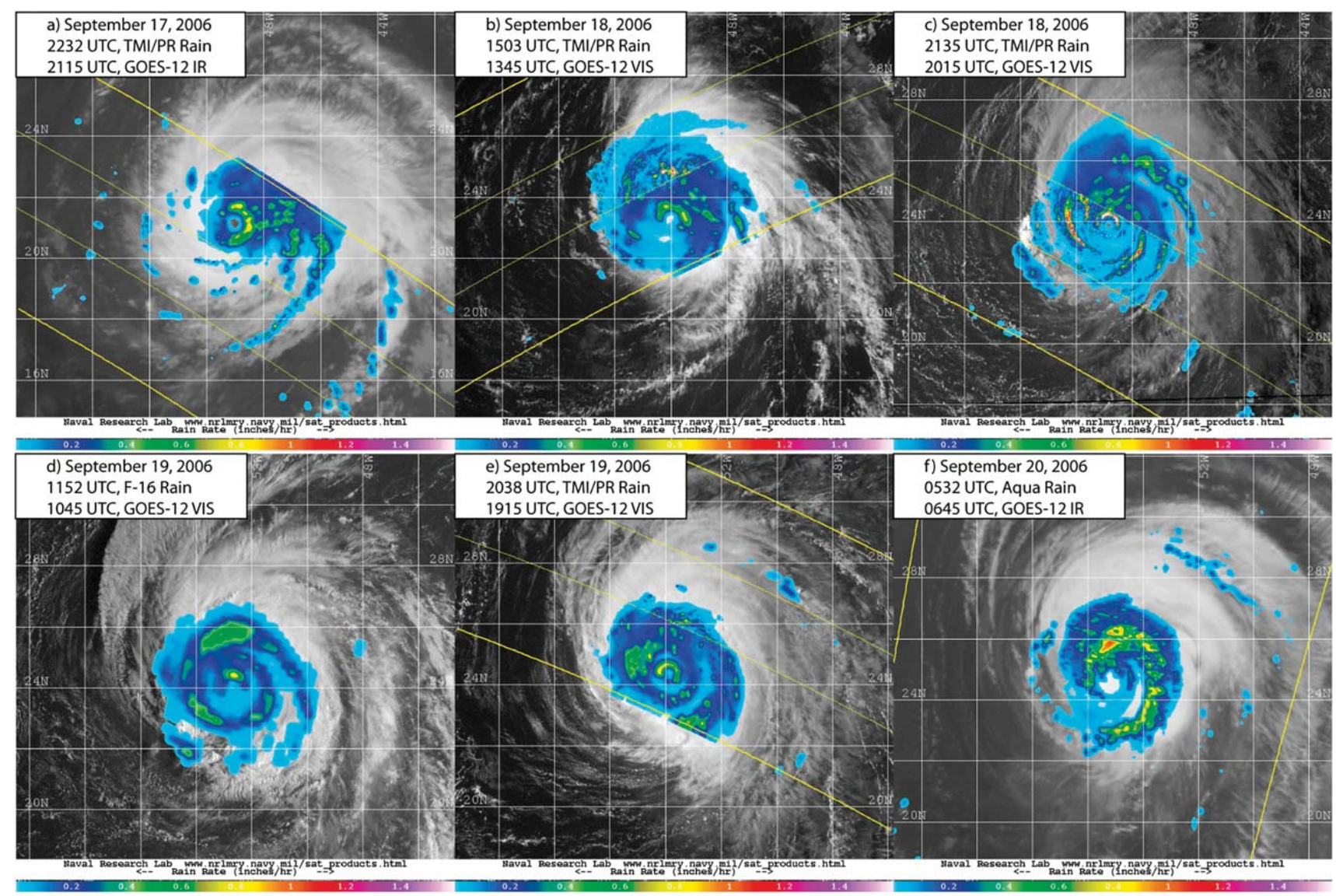

FIG. 19. GOES infrared or visible imagery and passive microwave rainfall estimates for various times during an eyewall replacement cycle during 17-20 Sep. Microwave imagery were provided by the Naval Research Laboratory tropical cyclone website.

near $600 \mathrm{hPa}$. Warm, stable air associated with the SAL lay north of the AEJ where convection was suppressed while cooler, more unstable air was south of the jet and readily produced convective systems on the cyclonic shear side of the jet. Winds in the bulk of the dust layer above the boundary layer were oriented primarily tangential to the vortex so that much of the dust was advected westward away from the storm by 15 September. This midlevel SAL air mass was gradually replaced by a distinct subsidence layer that led to marked drying on the northern and western sides of the storm.

QuikSCAT winds on 12 September suggested a very broad surface cyclone when the disturbance first moved off of Africa. AIRS, AMSR-E, and global meteorological analysis data on the same day indicated organized linear convective systems within a moist envelope or pouch delineated by closed streamlines in a reference frame moving with the storm (Dunkerton et al. 2009). There was likely some entrainment of dusty air into the convection at low levels as a result of low-level $(850-500 \mathrm{hPa})$ easterly wind shear, but this inflow likely occurred primarily in the moist boundary layer. At midlevels, where the pouch was stronger and inflow was weak, dry SAL air generally did not penetrate within $\sim 300 \mathrm{~km}$ of the center. These observations imply that the dry air may have enhanced some downdrafts on the northern- and westernmost fringes of the storm, but idealized simulations by Braun et al. (2012) suggest that the impact on storm intensity would have been only a small slowing or delay of intensification. Above the SAL, dry air penetrated farther in toward the storm center, but the impact of this dry air is uncertain.

During the intensification to major hurricane, dry air wrapped around the storm, with evidence that it wrapped completely around the eastern side and back around to the northern and western sides. Despite the dry air, the storm underwent steady intensification, including a period of rapid intensification, and formed a well-defined eyewall and outer rainbands. Dust was limited to the lowest layers $(<2 \mathrm{~km})$ on the southern side of the storm. Very dry air occurred through a deep layer above the boundary layer, and according to trajectory analyses was related to large-scale subsidence. Given the weak wind shear and strong intensification during this period, the dry air likely had only a minor braking influence on intensification. 

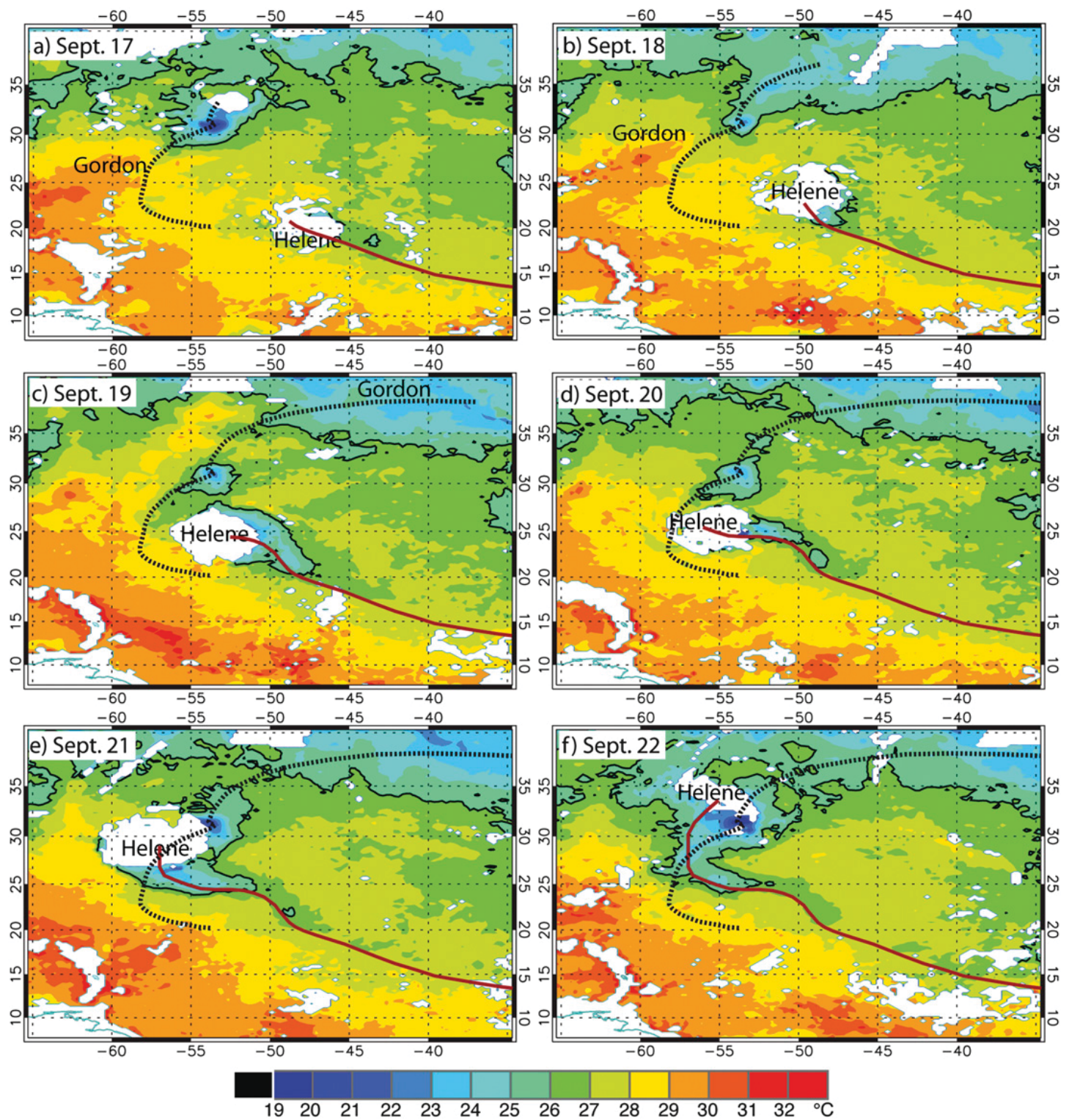

FIG. 20. Daily averaged sea surface temperature from all TRMM and AMSR-E orbits for the indicated dates. The thin black contour indicates the $26^{\circ} \mathrm{C}$ contour. The track of Hurricanes Gordon and Helene are indicated by the black dotted and red solid lines, respectively, for times prior to 1200 UTC on the indicated date.

The large-scale deep-layer vertical wind shear was very weak during the early development phase, increased to $4-6 \mathrm{~m} \mathrm{~s}^{-1}$ during the intensification stage, but did not exceed $6 \mathrm{~m} \mathrm{~s}^{-1}$ until the time of extratropical transition, approximately 2 days after Helene had begun to weaken. This timing suggests that vertical wind shear was not responsible for the weakening of Helene on 18 September. Instead, storm weakening was attributed to two factors. First, Helene underwent an eyewall replacement cycle near the time of weakening. This process may account for the sudden end to intensification, but probably does not explain the subsequent gradual weakening. Second, as Helene strengthened, its forward motion slowed considerably. Concurrent with this slowing down, there was an increase in the magnitude and scale of a cold wake that likely reduced the energy available to the storm. The combination of the eyewall replacement cycle and the ocean feedback was most likely responsible for the weakening of the storm.

Here we have focused primarily on the role of the dry air and higher stability associated with the SAL. It is difficult to fully assess the role of the SAL during the very early stages of Helene's formation (12-15 September) without detailed modeling, including the impacts of dust. The intensification of the storm after the departure of the SAL air mass on 15 September would appear to be 
consistent with the findings of DV04 of a possible suppressing influence on storm development during early stages, but the satellite data do not provide compelling evidence for an intrusion of dry, stable SAL air into the core of the vortex that might have disrupted development. Instead, the QuikSCAT data suggest that the slow intensification between 12 and 15 September might have resulted from relatively slow gestation processes associated with the initially broad surface circulation. However, satellite and airborne data are rather incomplete in space and time, and the interpretation of the data is inherently subjective. This topic may be examined in a future study using high-resolution simulations of Helene.

While the presence of the African easterly jet during early stages did not significantly increase the large-scale deep-layer vertical wind shear as defined in this study, it did produce low-level $(850-500 \mathrm{hPa})$ easterly vertical wind shear during the first two days of development (12-13 September) when intensification was slow. However, it is difficult to determine from the global analyses whether the AEJ was responsible for this slower storm development since Ross et al. (2009) argued that the AEJ actually contributed to disturbance growth via barotropic energy conversions during the early stages of Helene. Therefore, further work on the role of the jet is warranted.

Acknowledgments. We thank John Knaff and two anonymous reviewers for their comments on the manuscript. This work was supported by Dr. Ramesh Kakar at NASA Headquarters with funds from the NASA Hurricane Science Research Program. The TRMM and AMSR-E SST data were produced by Remote Sensing Systems who has been sponsored by NASA Earth Science programs. LASE data were provided by Drs. Ed Browell, Richard Ferrare, and Syed Ismail from NASA Langley Research Center.

\section{REFERENCES}

Bender, M. A., and I. Ginis, 2000: Real-case simulations of hurricane-ocean interaction using a high-resolution coupled model: Effects on hurricane intensity. Mon. Wea. Rev., 128, 917-946.

- - - and Y. Kurihara, 1993: Numerical simulations of tropical cyclone-ocean interaction with a high-resolution coupled model. J. Geophys. Res., 98 (D12), 23 245-23263.

Berry, G. J., and C. D. Thorncroft, 2012: African easterly wave dynamics in a mesoscale numerical model: The upscale role of convection. J. Atmos. Sci., 69, 1267-1283.

Brand, S., 1971: The effects on a tropical cyclone of cooler surface waters due to upwelling and mixing produced by a prior tropical cyclone. J. Appl. Meteor., 10, 865-874.

Braun, S. A., 2010a: Re-evaluating the role of the Saharan Air Layer in Atlantic tropical cyclogenesis and evolution. Mon. Wea. Rev., 138, 2007-2037.
_ 2010b: Comments on "Atlantic tropical cyclogenetic processes during SOP-3 NAMMA in the GEOS-5 Global Data Assimilation and Forecast System." J. Atmos. Sci., 67, 24022410.

—- and L. Wu, 2007: A numerical study of Hurricane Erin (2001). Part II: Shear and the organization of eyewall vertical motion. Mon. Wea. Rev., 135, 1179-1194.

_ J. A. Sippel, and D. S. Nolan, 2012: The impact of dry midlevel air on hurricane intensity in idealized simulations with no mean flow. J. Atmos. Sci., 69, 236-257.

Brennan, M. J., C. C. Hennon, and R. D. Knabb, 2009: The operational use of QuikSCAT ocean surface vector winds at the National Hurricane Center. Wea. Forecasting, 24, 621-645.

Burpee, R. W., 1972: The origin and structure of easterly waves in the lower troposphere of North Africa. J. Atmos. Sci., 29, $77-90$.

Carlson, T. N., and J. M. Prospero, 1972: The large-scale movement of Saharan air outbreaks over the northern equatorial Atlantic. J. Appl. Meteor., 11, 283-297.

Chelton, D. B., M. H. Freilich, J. M. Sienkiewicz, and J. M. Von Ahn, 2006: On the use of QuikSCAT scatterometer measurements of surface winds for marine weather prediction. Mon. Wea. Rev., 134, 2055-2071.

Dare, R. A., and J. L. McBride, 2011: Sea surface temperature response to tropical cyclones. Mon. Wea. Rev., 139, 3798 3808.

DeMaria, M., and J. Kaplan, 1994: A Statistical Hurricane Intensity Prediction Scheme (SHIPS) for the Atlantic basin. Wea. Forecasting, 9, 209-220.

_ , and _ 1999: An updated Statistical Hurricane Intensity Prediction Scheme (SHIPS) for the Atlantic and eastern North Pacific basins. Wea. Forecasting, 14, 326-337.

Dunion, J. P., 2011: Rewriting the climatology of the tropical North Atlantic and Caribbean Sea atmosphere. J. Climate, 24, 893908.

, and C. S. Velden, 2004: The impact of the Saharan air layer on Atlantic tropical cyclone activity. Bull. Amer. Meteor. Soc., 85, 353-365.

— , and C. S. Marron, 2008: A reexamination of the Jordan mean tropical sounding based on awareness of the Saharan Air Layer: Results from 2002. J. Climate, 21, 5242-5253.

Dunkerton, T. J., M. T. Montgomery, and Z. Wang, 2009: Tropical cyclogenesis in a tropical wave critical layer: Easterly waves. Atmos. Chem. Phys., 9, 5587-5646.

Emanuel, K. A., 1989: The finite-amplitude nature of tropical cyclogenesis. J. Atmos. Sci., 46, 3431-3456.

Frank, W. M., and E. A. Ritchie, 1999: Effects of environmental flow upon tropical cyclone structure. Mon. Wea. Rev., 127, 2044-2061.

— and structure of numerically simulated hurricanes. Mon. Wea. Rev., 129, 2249-2269.

Franklin, J. L., and D. P. Brown, 2008: Atlantic hurricane season of 2006. Mon. Wea. Rev., 136, 1174-1200.

Gray, W. M., 1968: Global view of the origin of tropical disturbances and storms. Mon. Wea. Rev., 96, 669-700.

Huffman, G. J., and Coauthors, 2007: The TRMM Multisatellite Precipitation Analysis (TMPA): Quasi-global, multiyear, combined-sensor precipitation estimates at fine scales. J. Hydrometeor., 8, 38-55.

Ismail, S., and Coauthors, 2010: LASE measurements of water vapor, aerosol, and cloud distributions in Saharan air layers and tropical disturbances. J. Atmos. Sci., 67, 1026-1047. 
Jenkins, G. S., and A. Pratt, 2008: Saharan dust, lightning and tropical cyclones in the eastern tropical Atlantic during NAMMA-06. Geophys. Res. Lett., 35, L12804, doi:10.1029/ 2008GL033979.

— - — , and A. Heymsfield, 2008: Possible linkages between Saharan dust and tropical cyclone rain band invigoration in the eastern Atlantic during NAMMA-06. Geophys. Res. Lett., 35, L08815, doi:10.1029/2008GL034072.

Jones, T. A., D. J. Cecil, and J. Dunion, 2007: The environmental and inner-core conditions governing the intensity of Hurricane Erin (2007). Wea. Forecasting, 22, 708-725.

Karyampudi, V. M., and T. N. Carlson, 1988: Analysis and numerical simulations of the Saharan air layer and its effect on easterly wave disturbances. J. Atmos. Sci., 45, 3102-3136.

— , and H. F. Pierce, 2002: Synoptic-scale influence of the Saharan air layer on tropical cyclogenesis over the eastern Atlantic. Mon. Wea. Rev., 130, 3100-3128.

—_ and Coauthors, 1999: Validation of the Saharan dust plume conceptual model using lidar, Meteosat, and ECMWF data Bull. Amer. Meteor. Soc., 80, 1045-1075.

Khain, A. P., and I. D. Ginis, 1991: The mutual response of a moving tropical cyclone and the ocean. Beitr. Phys. Atmos., 64, 125-142.

Knaff, J. A., M. DeMaria, C. R. Sampson, J. E. Peak, J. Cummings, and W. H. Schubert, 2013: Upper oceanic energy response to tropical cyclone passage. J. Climate, 26, 2631-2650.

Lau, K. M., and J. M. Kim, 2007a: How nature foiled the 2006 hurricane forecasts. Eos, Trans. Amer. Geophys. Union, 88 (9), 105-107.

- and - 2007b: Cooling of the Atlantic by Saharan dust Geophys. Res. Lett., 34, L23811, doi:10.1029/2007GL031538.

Lin, I.-I., I.-F. Pun, and C.-C. Wu, 2009: Upper-ocean thermal structure and the western North Pacific category 5 typhoons. Part II: Dependence on translation speed. Mon. Wea. Rev., 137, 3744-3757.

Merrill, R. T., 1988: Environmental influences on hurricane intensification. J. Atmos. Sci., 45, 1678-1687.

Messager, C., D. J. Parker, O. Reitebuch, A. Agusti-Panareda, C. M. Taylor, and J. Cuesta, 2010: Structure and dynamics of the Saharan atmospheric boundary layer during the West African monsoon onset: Observations and analyses from the research flights of 14 and 17 July 2006. Quart. J. Roy. Meteor. Soc., 136, 107-124.

Portabella, M., and A. Stoffelen, 2001: Rain detection and quality control of SeaWinds. J. Atmos. Oceanic Technol., 18, 11711183.

Powell, M. D., 1990: Boundary layer structure and dynamics in outer hurricane rainbands. Part II: Downdraft modification and mixed-layer recovery. Mon. Wea. Rev., 118, 918-938.

Price, J. F., 1981: Upper ocean response to a hurricane. J. Phys. Oceanogr., 11, 153-175.

Reale, O., and W. K. Lau, 2010: Reply. J. Atmos. Sci., 67, 24112415.

, - - K.-M. Kim, and E. Brin, 2009: Atlantic tropical cyclogenetic processes during SOP-3 NAMMA in the GEOS-5 global data assimilation and forecast system. J. Atmos. Sci., 66, 3563-3578.
Reed, R. J., D. C. Norquist, and E. E. Recker, 1977: The structure and properties of African wave disturbances as observed during phase III of GATE. Mon. Wea. Rev., 105, 317-333.

Riemer, M., and M. T. Montgomery, 2011: Simple kinematic models for the environmental interaction of tropical cyclones in vertical wind shear. Atmos. Chem. Phys., 11, 93959414

Rogers, R., S. Chen, J. Tenerelli, and H. Willoughby, 2003: A numerical study of the impact of vertical shear on the distribution of rainfall in Hurricane Bonnie (1998). Mon. Wea. Rev., 131, 1577-1599.

— , and Coauthors, 2006: The Intensity Forecasting Experiment: A multiyear field program for improving tropical cyclone intensity forecasts. Bull. Amer. Meteor. Soc., 87, 1523-1537.

Ross, R. S., T. N. Krishnamurti, S. Pattnaik, and A. Simon, 2009: Energy transformation and diabatic processes in developing and nondeveloping African easterly waves observed during the NAMMA project of 2006. Wea. Forecasting, 24, 15241548.

Rotunno, R., J. B. Klemp, and M. L. Weisman, 1988: A theory for strong, long-lived squall lines. J. Atmos. Sci., 45, 463-485.

Shu, S., and L. Wu, 2009: Analysis of the influence of the Saharan air layer on tropical cyclone intensity using AIRS/Aqua data. Geophys. Res. Lett., 36, L09809, doi:10.1029/2009GL037634.

Sippel, J. A., S. A. Braun, and C.-L. Shie, 2011: Environmental influences on the strength of Tropical Storm Debby (2006). J. Atmos. Sci., 68, 2557-2581.

Sun, D., K. M. Lau, and M. Kafatos, 2008: Contrasting the 2007 and 2005 hurricane seasons: Evidence of possible impacts of Saharan dry air and dust on tropical cyclone activity in the Atlantic basin. Geophys. Res. Lett., 35, L15405, doi:10.1029/ 2008GL034529.

,,--- Z. Boybeyi, G. Leptoukh, C. Yang, and R. Yang, 2009: Numerical simulations of the impacts of the Saharan Air Layer on Atlantic tropical cyclone development. J. Climate, 22, 6230-6250.

Tang, B., and K. Emanuel, 2012: A ventilation index for tropical cyclones. Bull. Amer. Meteor. Soc., 93, 1901-1912.

Vizy, E. K., and K. H. Cook, 2009: Tropical storm development from African easterly waves in the eastern Atlantic: A comparison of two successive waves using a regional model as part of NASA AMMA 2006. J. Atmos. Sci., 66, 3313-3334.

Wu, L., 2007: Impact of Saharan air layer on hurricane peak intensity. Geophys. Res. Lett., 34, L09802, doi:10.1029/ 2007GL029564.

- 2009: Comparison of atmospheric infrared sounder temperature and relative humidity profiles with NASA African Monsoon Multidisciplinary Analyses (NAMMA) dropsonde observations. J. Geophys. Res., 114, D19205, doi:10.1029/ 2009JD012083.

— S. A. Braun, J. J. Qu, and X. Hao, 2006: Simulating the formation of Hurricane Isabel (2003) with AIRS data. Geophys. Res. Lett., 33, L04804, doi:10.1029/2005GL024665.

Zipser, E. J., and Coauthors, 2009: The Saharan Air Layer and the fate of African easterly waves-NASA's AMMA field study of tropical cyclogenesis. Bull. Amer. Meteor. Soc., 90, 1137-1156. 